\title{
High photon count rates improve the quality of super-resolution
}

\section{fluorescence fluctuation spectroscopy}

Falk Schneider ${ }^{1 *}$, Pablo Hernandez-Varas ${ }^{2,3}$, B. Christoffer Lagerholm², Dilip Shrestha ${ }^{1,}$ Erdinc Sezgin $^{1,4}$, M. Julia Roberti ${ }^{5}$, Giulia Ossato ${ }^{5}$, Frank Hecht ${ }^{5}$, Christian Eggeling ${ }^{1,2,6,7}$, Iztok Urbančič1,8*

${ }^{1}$ MRC Human Immunology Unit and ${ }^{2}$ Wolfson Imaging Centre Oxford, MRC Weatherall Institute of Molecular Medicine, University of Oxford, Headley Way, Oxford, OX3 9DS, United Kingdom

${ }^{3}$ Core Facility for Integrated Microscopy, Panum Institute, University of Copenhagen, 2200 Copenhagen N, Denmark

${ }^{4}$ Science for Life Laboratory, Department of Women's and Children's Health, Karolinska Institutet, Stockholm, Sweden

${ }^{5}$ Leica Microsystems CMS GmbH, Am Friedensplatz 3, 68163 Mannheim, Germany

${ }^{6}$ Institute of Applied Optics and Biophysics, Friedrich-Schiller-University Jena, Max-Wien Platz 4, 07743 Jena, Germany

${ }^{7}$ Leibniz Institute of Photonic Technology e.V., Albert-Einstein-Straße 9, 07745 Jena, Germany

${ }^{8}$ Jožef Stefan Institute, Jamova cesta 39, SI-1000 Ljubljana, Slovenia

* corresponding authors (email: falk.schneider@rdm.ox.ac.uk, iztok.urbancic@ijs.si)

Keywords: FCS, STED, high count rates, diffusion 


\section{Abstract}

Probing the diffusion of molecules has become a routine measurement across the life sciences, chemistry and physics. It provides valuable insights into reaction dynamics, oligomerisation, molecular (re-)organisation or cellular heterogeneities. Fluorescence correlation spectroscopy (FCS) is one of the widely applied techniques to determine diffusion dynamics in two and three dimensions. This technique relies on the temporal autocorrelation of intensity fluctuations but recording these fluctuations has thus far been limited by the detection electronics, which could not efficiently and accurately time-tag photons at high count rates. This has until now restricted the range of measurable dye concentrations, as well as the data quality of the FCS recordings, especially in combination with super-resolution stimulated emission depletion (STED) nanoscopy.

Here, we investigate the applicability and reliability of (STED-)FCS at high photon count rates (average intensities of up to $40 \mathrm{MHz}$ ) using novel detection equipment, namely hybrid detectors and real-time gigahertz sampling of the photon streams implemented on a commercial microscope. By measuring the diffusion of fluorophores in solution and cytoplasm of live cells, as well as in model and cellular membranes, we show that accurate diffusion and concentration measurements are possible in these previously inaccessible high photon count regimes. Specifically, it offers much greater flexibility of experiments with biological samples with highly variable intensity, e.g. due to a wide range of expression levels of fluorescent proteins. In this context, we highlight the independence of diffusion properties of cytosolic GFP in a concentration range of approx. 0.01-1 $\mu \mathrm{M}$. We further show that higher photon count rates also allow for much shorter acquisition times, and improved data quality. Finally, this approach also pronouncedly increases the robustness of challenging live cell STED-FCS measurements of nanoscale diffusion dynamics, which we testify by confirming a free diffusion pattern for a fluorescent lipid analogue on the apical membrane of adherent cells. 


\section{Introduction}

Fluorescence correlation spectroscopy (FCS) has, since its introduction almost 50 years ago, become a widely applied technique to study diffusion dynamics in synthetic and biological applications ${ }^{1-3}$. It has greatly contributed to the understanding of molecular diffusion in model systems and living cells, both in 2D (in vitro models or cellular membranes) and in 3D (solution or cellular cytoplasm and nucleus) environments ${ }^{4-8}$. Notably, it has offered fundamental insights into the dynamic organisation of living systems at the molecular level, e.g. by characterising the transient, dynamic, yet structured nature of the organisation of fluid membranes ${ }^{4,5,9}$.

FCS provides a plethora of information about molecular dynamics. The diffusion rates and local concentrations of fluorescent molecules can be determined directly from the autocorrelation functions $s^{1,10,11}$. The spatial variability can be further evaluated by laser-scanning ${ }^{12-14}$ and imagingbased variants of $\mathrm{FCS}^{15-18}$. Further, molecular interactions can be probed either directly, e.g. binding of molecules detected by cross-correlation $(\mathrm{FCCS})^{19}$, or indirectly via variations of the apparent diffusion coefficient at different length scales, measured by spot-variation $\mathrm{FCS}^{20}$ providing information on diffusion modes as in single particle tracking ${ }^{21}$. Finally, the combination of FCS with superresolution stimulated emission depletion (STED) microscopy allows direct observation of nanoscale diffusion dynamics, shedding new light on molecular organisation below the diffraction limit ${ }^{22}$.

All these invaluable details are extracted from intensity fluctuations due to the transit of fluorescent molecules through the observation spot of the microscope. As the fluctuations (i.e. bursts in the fluorescence intensity trace) are most obvious for sparsely labelled samples, FCS is often considered a single molecule technique, and has thus been shown multiple times to perform accurately in the range of pico- and nanomolar concentrations ${ }^{2}$. These concentrations, though, can be far from physiological levels present in living systems, where molecular abundance can be much higher (e.g. average concentration of a protein in eukaryotic yeast cells is estimated to be around $1 \mu \mathrm{M}^{23}$ ). Nevertheless, it has been theoretically predicted and experimentally verified that FCS can perform 
similarly and can generate accurate results also for much larger concentrations $(>100 \mathrm{nM})^{24}$. In this regime, the main factors for signal quality of FCS, often described by the signal-to-noise ratio (SNR), are the acquisition time $(T)$, and the number of detected photons per molecule (i.e. molecular brightness, $B$, which depends on the absorption cross section and quantum yield of the dye, the power of the excitation laser, and the detection efficiency of the measurement setup): SNR $\propto B \times T^{1 / 2}$ (see for example $11,25-28)$.

For the most efficient and reliable detection of fluorescence fluctuations, sensitive single-photoncounting detectors are typically used, often coupled to fast electronics that enable accurate recording of photon arrival times thus also allowing additional photon filtering in post-processing ${ }^{29}$. One of the main drawbacks of this instrumentation, however, has been its rather long dead time after each photon detection $(>100 \mathrm{~ns})^{30}$, limiting photon count rates to a few $\mathrm{MHz}$, which is far lower than the typical repetition rate of excitation lasers running at $20-80 \mathrm{MHz}$. This has posed a severe limitation to the accuracy and flexibility in FCS experiments at high fluorophore concentrations, which are however unavoidable for many applications - for example when measuring binding dynamics of low affinity, or diffusion dynamics and concentrations of cellular proteins at different expression levels. Several approaches have been developed to enable FCS measurements even in such cases: labelling of only a fraction of the molecules, reduction of the simultaneously visible fluorophores via fluorescence photoswitching ${ }^{31,32}$, splitting-up of the signal onto several detectors such as on custom-built detector banks $s^{33}$, or reduction of the effective observation volume ${ }^{34,35}$ using for example small sample containers $^{36}$, near-field structures ${ }^{37,38}$, plasmonic near-field optics ${ }^{39-41}$, or super-resolution STED microscopy ${ }^{5,42}$. Unfortunately, all of these techniques introduce more complexity and possible bias, for example due to required controls to check whether the fraction of labelled or photoswitched molecules truly reflects the entire population, influence on the sample and fluorescent molecules by surface or small volume effects, setup complexity, or perplexing photophysics of the fluorescent label. 
Here, we demonstrate the straightforward realisation of FCS measurements at high photon count rates on a commercially available microscope, using novel photon counting instrumentation. By measuring the diffusion of fluorescent dyes in solutions, artificial and cell membranes, we explore performance, capabilities, accuracy, applicability and limitations of confocal FCS and STED-FCS experiments at photon count rates of up to $20-30 \mathrm{MHz}$ per detection channel, revealing great potential for FCS experiments at high count rates. As examples, we show the independence of cytosolic protein diffusion on cellular expression levels and the application of high count rates to STEDFCS measuring the diffusion behaviour of lipids in apical cellular plasma membranes.

\section{Results and Discussion}

Non-saturated photon detection at high dye concentrations and laser excitation powers

We first tested the advanced photon counting instrumentation, implemented on a confocal and STEDcapable microscope, by recording fluorescence fluctuation data from a single dye (Atto655, chosen for its low population of triplet states) diffusing in aqueous solution at different concentrations or excitation laser powers, resulting in different photon count rates. The photon counting instrumentation included hybrid detectors with very short dead times and fast FPGA electronics with real-time $\mathrm{GHz}$ sampling and pattern matching, which together with the $80 \mathrm{MHz}$ pulsed fluorescence excitation allows for detection of photon count rates of tens of $\mathrm{MHz}$ without corrections (as described in detail in the Materials and Methods section). Figure $1 \mathrm{~A}$ and $\mathrm{B}$ show fluctuations in the normalized photon count rates over time as recorded for three different dye concentrations and laser excitation powers, respectively. As expected from theory ${ }^{10,25-28}$, the relative fluctuations around the average count rate decrease with increasing dye concentration, but much less so with excitation laser power. Most importantly, we could follow a linear increase of photon count rate with dye concentration and laser excitation power (Figure $1 \mathrm{C}$ and $\mathrm{D}$ ), as expected in the absence of limitations in detection electronics. Note that approximate linearity was maintained despite employing dye concentrations of 
up to $1 \mu \mathrm{M}$ and registering photon count rates of up to $20-30 \mathrm{MHz}$. The non-linearity introduced at excitation laser powers larger than $40 \mu \mathrm{W}\left(\approx 30 \mathrm{~kW} / \mathrm{cm}^{2}\right.$, Figure 1D) were to be expected due to dye photobleaching and saturation of excited state population and consequently fluorescence emission (i.e. not due to detector saturation, compare Figure $1 \mathrm{C}$ at same count rate levels), while slight saturation effects at very high dye concentrations may result from photon re-absorption and dye selfquenching, as indicated previously ${ }^{33,43}$. In due consideration of acquisition count rate being the limiting factor in conventional equipment, we present the rest of the data as a function of this parameter.

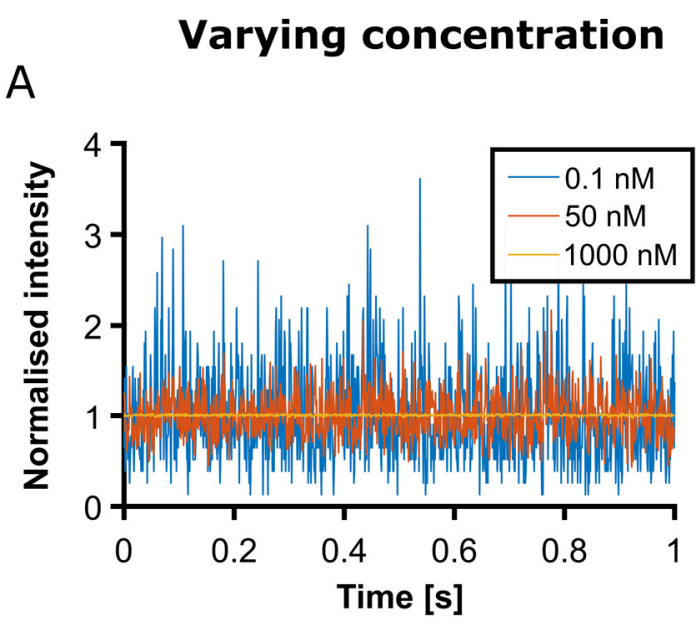

C

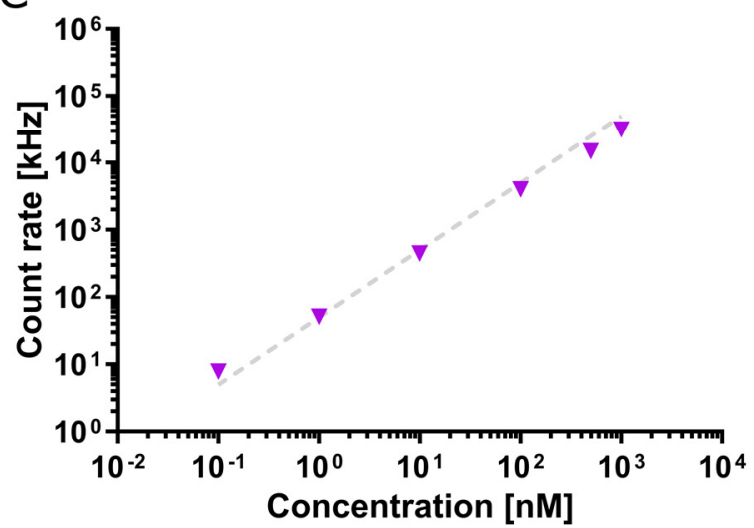

\section{Varying excitation power}

B

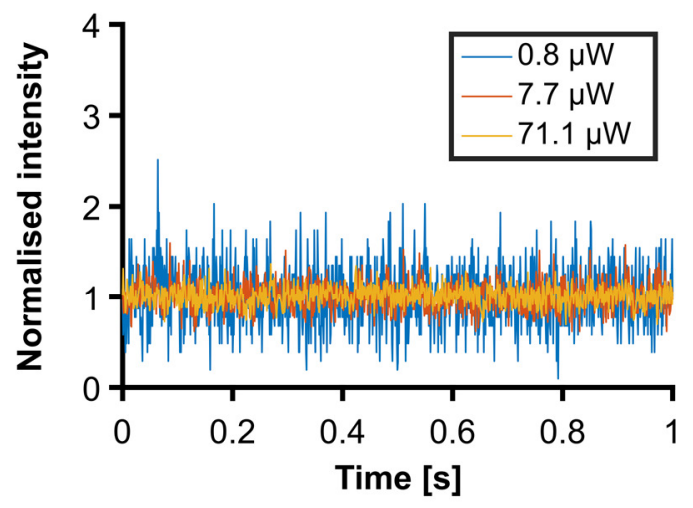

D

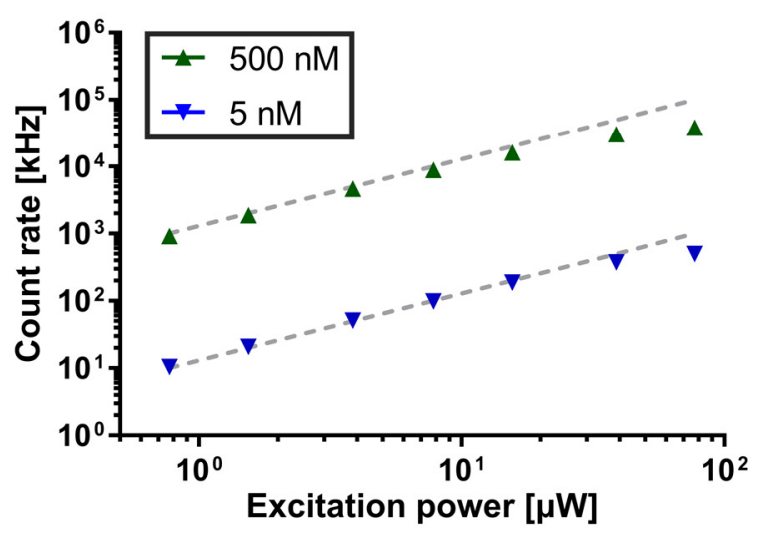

Figure 1: Influence of varying concentration and excitation laser power on FCS measurements of the dye Atto655 in aqueous solution. $\mathbf{A}, \mathbf{B}$ Normalized detected photon count rate data (intensity) over time at different dye concentrations ( $\mathrm{A}, 0.1 \mathrm{nM}, 50 \mathrm{nM}$, and $1 \mu \mathrm{M}$ as labelled; excitation laser power $15.4 \mu \mathrm{W}$ ) and excitation laser powers ( $B$, as labelled; concentration $5 \mathrm{nM}$ ), highlighting the reduction in relative intensity fluctuations for higher concentrations and excitation laser powers. C,D Detected photon count rates versus instituted dye concentration ( $C$, dashed lines indicate the expected linear increase; excitation laser power $15.4 \mu \mathrm{W}$ ) and 
instituted excitation laser power for $5 \mathrm{nM}$ (blue) and $500 \mathrm{nM}$ (green) (D, dashed lines indicate the expected linear increase ). Values are averages of three repetitions (acquisition time $15 \mathrm{~s}$ each), and standard deviations are smaller than the size of the symbols.

FCS noise levels at different count rates

The ability to record photon time traces at high count rates consequently allowed us to acquire FCS data for Atto655 up to $1 \mu \mathrm{M}$ high dye concentrations and excitation laser powers up to $40 \mu \mathrm{W}$ $\left(\approx 30 \mathrm{~kW} / \mathrm{cm}^{2}\right)$. The autocorrelation curves for these unconventional conditions show similar decays as for low dye concentrations and laser powers (Figure 2A and B). From common FCS theory (assuming large particle concentrations and excluding non-linear photo-physical effects such as saturation and photobleaching), FCS-derived average transit times should be independent of dye concentration and laser power, while the amplitude of the autocorrelation curve should linearly decrease with dye concentration and stay constant with laser power. We could well recover this behaviour from fitting our FCS data, even when recorded at count rates of up to $20-30 \mathrm{MHz}$ (Figure $2 \mathrm{C}$ and $\mathrm{D}$ ). As before, deviations at the highest tested laser powers $>40 \mu \mathrm{W}$ (primarily apparent as a drop in the values of the transit time and FCS amplitudes, Figure 2D), can be attributed to dye photobleaching and fluorescence emission saturation. For dyes other than Atto655, excessive pumping into their triplet states would result in additional deterioration at high excitation powers and would thus need to be carefully considered as well ${ }^{44,45}$.

An interesting feature of the FCS data recorded at different dye concentrations or excitation laser powers, i.e. photon count rates, are the different noise levels and resulting data quality. From theory ${ }^{11,24,26}$, the noise in FCS data should linearly decrease with increasing excitation laser power and be independent of dye concentration. The latter has for example been experimentally verified for dye concentrations of up to around $100 \mathrm{nM}^{24}$. Consequently, we set out to investigate noise levels for FCS data recorded at the large dynamic range of dye concentrations and laser powers, which became accessible using the new equipment. 

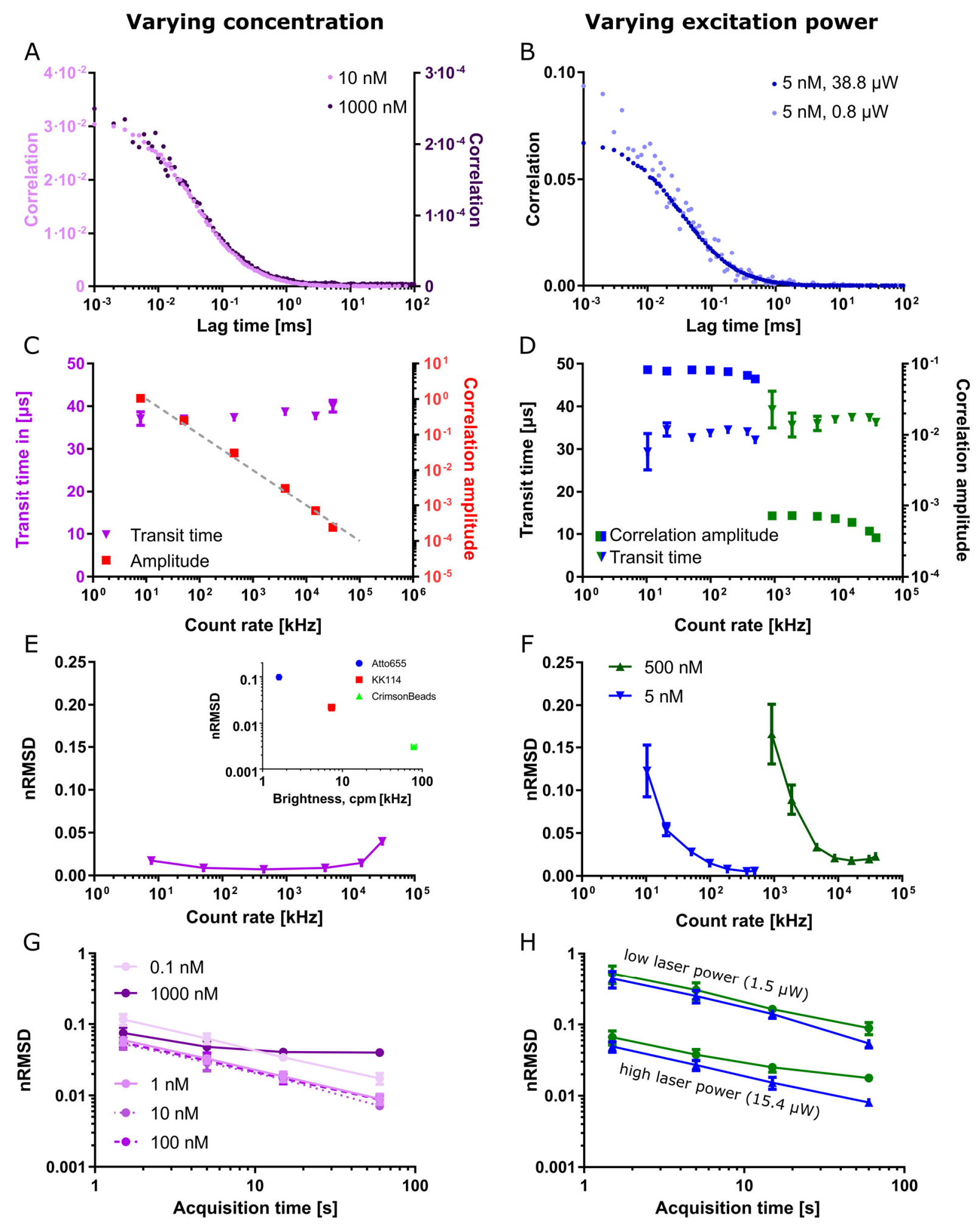

Figure 2: Detailed analysis of the FCS data of Atto655 in aqueous solution at varying instituted dye concentration $C_{\text {dye }}$ (left panels) and excitation laser power Plaser (right panels). A,B Representative FCS curves at different concentrations ( $A$, as labelled, $P_{\text {laser }}=15.4 \mu \mathrm{W}$ ) and excitation laser powers $\left(B\right.$, as labelled, $\left.C_{d y e}=5 \mathrm{nM}\right)$. C-F Transit time, correlation amplitude ( $C$ and $D$, axes and colours as labelled, $D$ : for two $C_{\text {dye }}$ as labelled), and nRMSD (i.e. noise in correlation data, $\mathrm{E}$ and F; F: for two $\mathrm{c}_{\text {dye }}$ as labelled) as determined from FCS for different photon count rates, i.e. different $C_{d y e}(C, E)$ and $P_{\text {laser }}(D, F)$. (Inset E) Values of nRMSD as determined from FCS for 
different dyes in aqueous solution with different brightness, i.e. counts-per-molecule (cpm) (average over $c_{\text {dye }} \approx$ 0.01-1 $\mu \mathrm{M}$ or 1:100-1:2000 dilutions of the stock bead suspension (see Figure SI 3), Plaser $=1.5 \mu W$ ). G,H Values of nRMSD as determined from FCS data of Atto655 at different acquisition times and at varying $c_{\text {dye }}\left(G, P_{\text {laser }}=\right.$ $15.4 \mu \mathrm{W})$ and $\mathrm{P}_{\text {laser }}\left(\mathrm{H}, \mathrm{C}_{\mathrm{dye}}=5\right.$ or $\left.500 \mathrm{nM}\right)$ as labelled. Data points represent averages and standard deviations (unless smaller than the symbols) of three repetitions (60 s acquisition time if not indicated otherwise).

Already visual inspection of our FCS data recorded at the different conditions (Figure 2A and B) indicated notable differences in noise levels (shown as the spread of the correlation curve), especially for the different excitation laser powers, which was most pronounced at short lag times (in this case up to around $40 \mu \mathrm{s}$, roughly corresponding to the transit time of the dye). The non-trivial estimation of noise levels in FCS data has been the subject of intense investigations ${ }^{11,24-29}$. In our experience, the noise levels in the current FCS data were well estimated by the root-mean-square of the fitting residuals normalized to the amplitude (nRMSD, calculated for short lag times up to the transit time), with lower values indicating better data quality (note that nRMSD was not used as the residuals minimisation metric in our fitting protocol; see Materials and Methods and Figure SI 1 for details). The nRMSD provided us with a single value for the data quality of each measurement. Conveniently, this measure also roughly corresponded to the relative standard deviation of the values of the average transit time as determined from fitting of the data (Figure SI 2, the nRMSD relates closely to the measurement error, i.e. the experimental standard deviation of the correlation at every data point, Figure SI 1). In accordance with the theoretical predictions ${ }^{11,24,26}$, the noise in the FCS data and thus nRMSD were only weakly affected by varying concentration (Figure 2E), but could be greatly improved by increased excitation laser power (Figure 2F). The excitation laser power directly increases the dyes' excited state population and thus fluorescence emission rate and the average detected count rate per single dye (molecular brightness), which is the reason for the improvements in noise levels. Under comparable measurement conditions, the nRMSD is therefore also a direct indicator of the molecular brightness of the investigated dye (inset in Figure $2 \mathrm{E}$ and Figure SI 3). Deteriorated noise levels, i.e. higher nRMSD values, were again observed at dye concentrations around $1 \mu \mathrm{M}$, but were much less 
pronounced at the highest excitation laser powers above $20-40 \mu \mathrm{W}$, despite saturation of photon count rates due to photophysical limitations of the dye (Figure 1D) and deviations of values of the transit times and correlation amplitudes (Figure 2D) as highlighted above.

\section{FCS noise levels at different acquisition times}

Predicted from theory and to a certain extent verified experimentally ${ }^{11,24,26}$, the noise in FCS data should decrease with the square root of the acquisition time. We could well reproduce this dependence for different dye concentrations and excitation laser powers (Figure $2 \mathrm{G}$ and $\mathrm{H}$; again, the same issues as outlined above caused deviations at high dye concentrations and excitation laser powers). This data establishes unique possibilities of adapting to experimental conditions. Due to the absence of saturation effects in photon count rates in the 5-500 nM concentration range, the possibility of increasing the laser power and detecting correspondingly higher photon count rates does not only increase the data quality (i.e. lower nRMSD values), but alternatively allows for a significant reduction (up to two orders of magnitude) in the acquisition time required for generating similar data quality (Figure $2 \mathrm{G}$ and $\mathrm{H}$, Figure SI 2).

FCS of cytosolic GFP in live cells at various expression levels

The possibility of acquiring accurate FCS data in a wide range of dye concentrations allows simplification or realization of experiments under challenging conditions. For example, FCS-based measurements of diffusion or concentration of fluorescent proteins in cells are usually challenged by the naturally varying expression levels of the fluorescent proteins, as shown in the representative confocal image in Figure 3A for HeLa cells expressing cytoplasmic GFP (green fluorescent protein). Using standard FCS instrumentation, only carefully chosen dim cells would be measurable, which may 
represent only a small, and not necessarily representative fraction of all cells, introducing a potential source of bias.

A
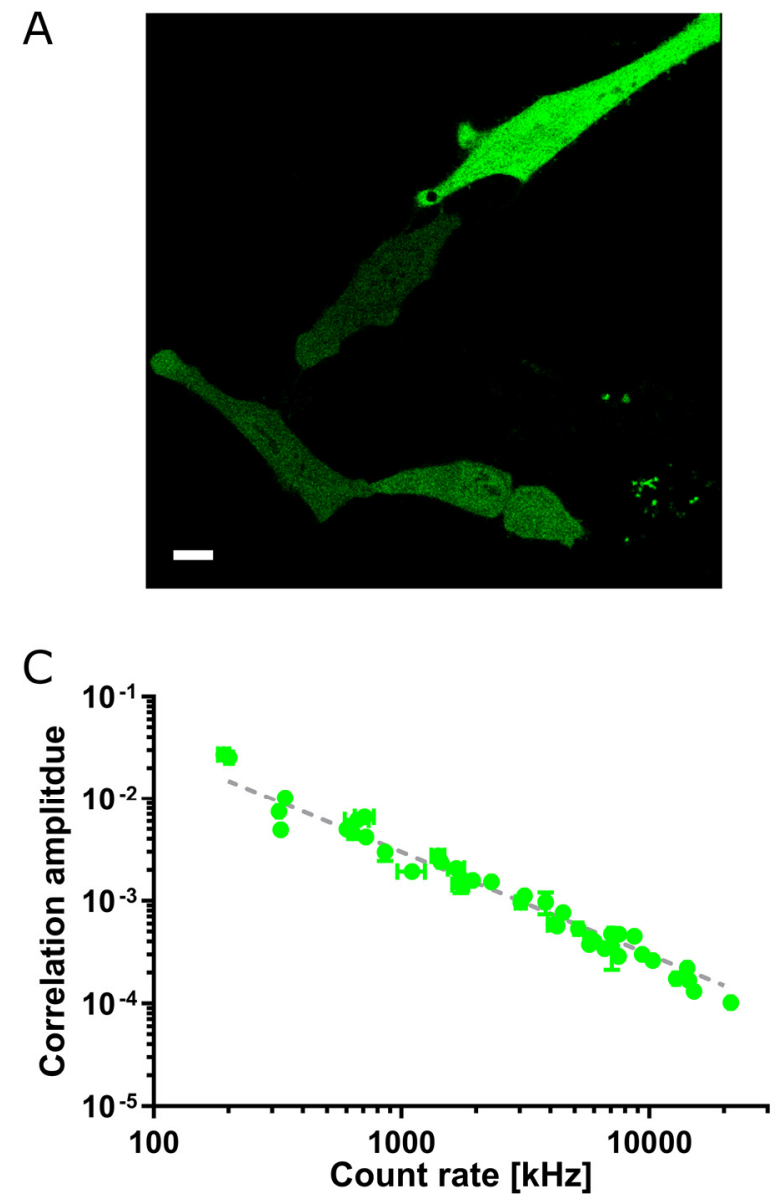

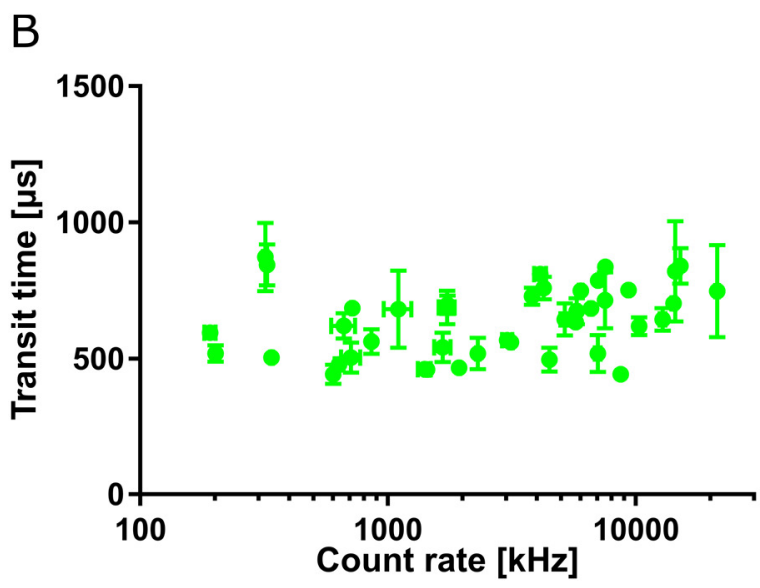

D

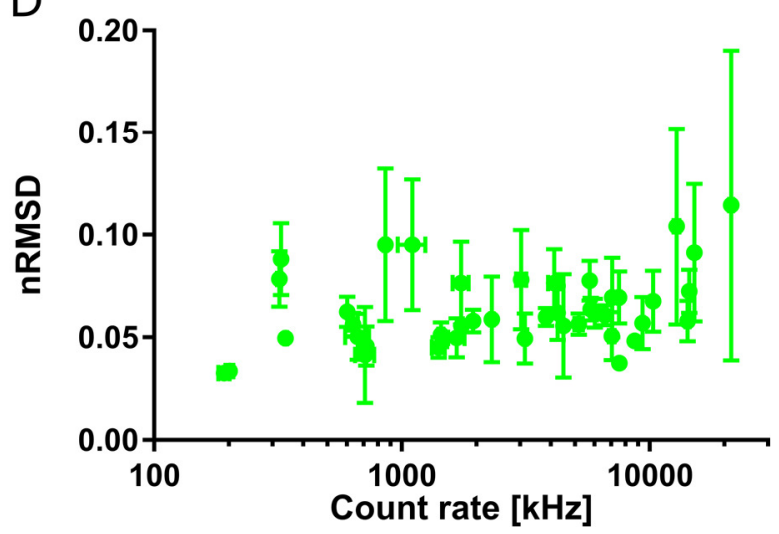

Figure 3: Acquiring FCS data in live cells at different expression levels of the cytosolic fluorescent protein GFP-

SNAP. A Representative confocal image of HeLa cells transiently transfected with cytoplasmic GFP-SNAP, highlighting different brightness and thus expression levels. Scale bar: $10 \mu \mathrm{m}$. B-D Values of transit time (B), correlation amplitude ( $C$, with the grey guideline indicating the predicted inverse relationship), and nRMSD (i.e. noise in correlation data, D) as determined from FCS for different photon count rates, originating from different expression levels. Averages and standard deviations of at least three measurements per cell (acquisition time $15 \mathrm{~s}$, excitation laser power $0.6 \mu \mathrm{W})$.

Using our current setup, we could now record FCS data for all HeLa cells irrespective of their fluorescence intensity, revealing average transit times of GFP over a wide range of photon count rates and thus concentrations resulting from different expression levels (Figure 3B). The photon count rates 
are correlated with the correlation amplitude (Figure 3C), which is inversely proportional to the average number of fluorescent molecules in the observation volume (see Materials and Methods) and thus concentration and expression level of GFP can be inferred. Taking our observation volume of about $1 \mathrm{fL}$, we can estimate concentrations of GFP of approx. $0.01-5 \mu \mathrm{M}$ between the differently expressing cells (see Materials and Methods). These data indicate that within the tested range the mobility of GFP is independent of expression level. In addition, the quality of the FCS data as quantified by the nRMSD values was maintained over the range of tested expression levels (Figure 3D), as predicted from the behaviour of the organic dye in solution (compare Figure 2). Only in the regime beyond $10 \mathrm{MHz}$ (in our case corresponding to concentrations around $1 \mu \mathrm{M}$ ), we observed a slight signal deterioration due to, for example, possible out-of-focus and self-absorption contributions, reflected in an increase of the nRMSD (Figure 3D) and a decrease in the amplitude beyond the predicted inverse relationship with the count rate (grey dashed guide line in Figure 3C).

\section{STED-FCS of lipid dyes in model membranes}

The main strength of FCS on a super-resolution STED microscope, STED-FCS, is the ability to directly report on nanoscale molecular mobility and thus determine apparent values of diffusion coefficients from the average transit times (see Materials and Methods) for different observation spot sizes - from conventional confocal spot sizes with lateral diameters of around $200 \mathrm{~nm}$, down to STED microscopy recordings with observation spot diameters of $30-40 \mathrm{~nm}$. From the dependency of the apparent values of the diffusion coefficient on the observation spot diameter, STED-FCS has provided insights into the molecular diffusion modes, similar to spot-variation $\mathrm{FCS}^{20}$, but now at the relevant molecular scale, which is particularly valuable for the elucidation of the nanoscale architecture of biological membranes ${ }^{22}$. However, measurements at various sizes of the effective observation spot inherently impose a large variation in the average number of fluorescent molecules in the observation spot $(N)$ and thus detected photon count levels (Figure 4A, schematics). Large observation spots at the confocal 
recordings entail already high count rates and high values of $N$ at rather low dye concentrations, while the smaller observation spots at the STED microscopy recordings require relatively large dye concentrations to reach photon count rates and values of $N$ that are high enough for allowing reasonably low acquisition times (it has also been shown theoretically that too low count rates or concentrations lead to noisy and inaccurate FCS data $\left.{ }^{11,24,26}\right)$. This has limited the range of useful dye concentrations in STED-FCS measurements using conventional detection electronics.
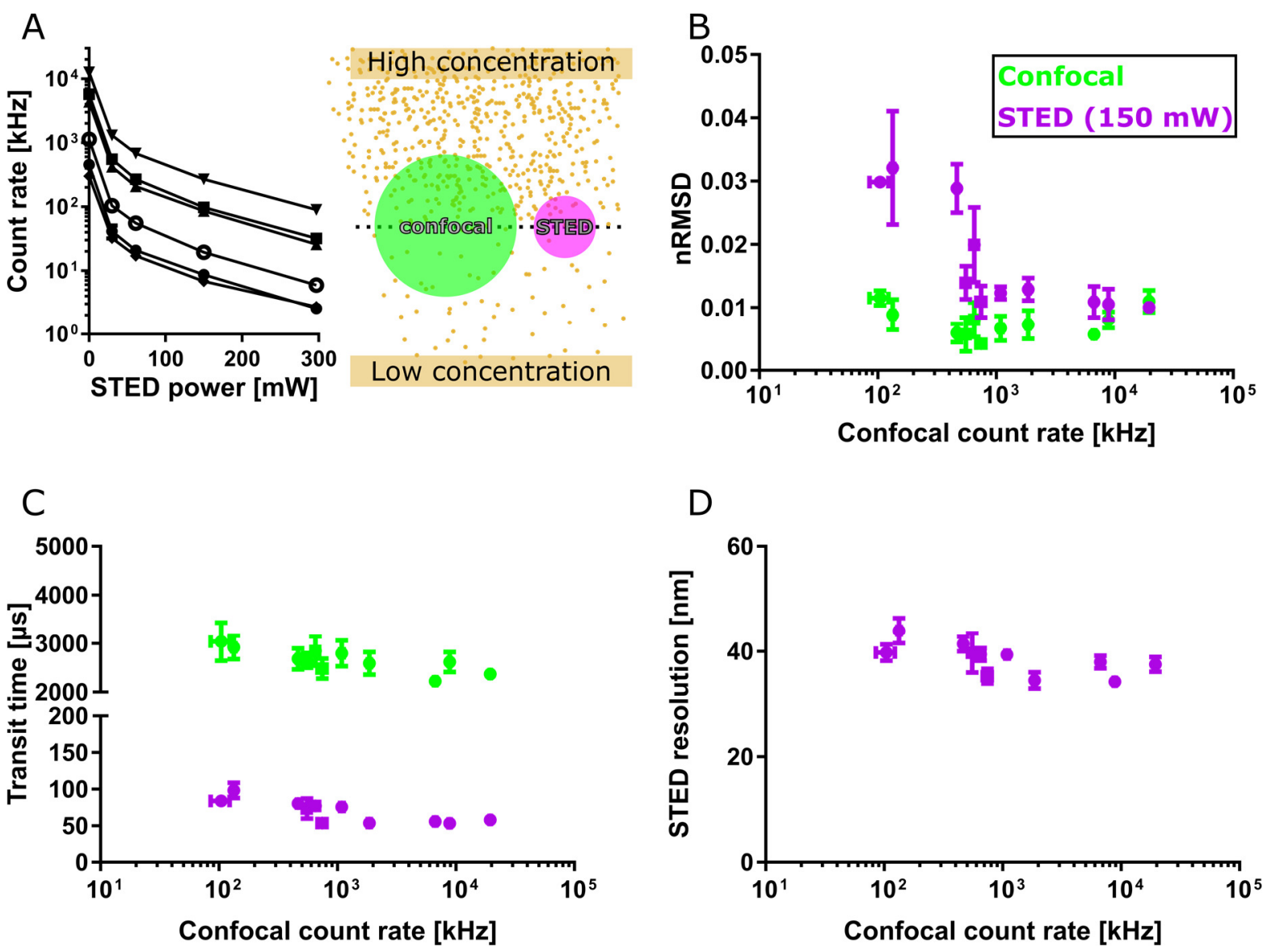

Figure 4: STED-FCS in supported lipid bilayers (SLBs) at various dye concentrations. A right Sketch illustrating the shrinking of the observation spot from the confocal (green) to the STED (purple) modus for a high or low concentration of dye molecules (yellow dots). Left Average fluorescence intensity experimentally recorded for different concentrations of a fluorescent lipid analogue (Abberior STAR Red-DPPE; different symbols) in DOPC SLBs for increasing powers of the STED laser. From these data, the noise level of FCS data (nRMSD, B), the average fitted transit time (C), and calculated apparent STED resolution (D) for confocal (green) and STED-FCS recordings (purple, $150 \mathrm{~mW}$ depletion power) are plotted against the respective confocal acquisition count rates, which are the limiting factor in each STED-FCS experiment. Excitation power $2.3 \mu \mathrm{W}$, acquisition time $15 \mathrm{~s}$. 
Each data-point represents the average and standard deviation of at least three measurements per SLB preparation.

To evaluate the performance of the new detection electronics in STED-FCS, we recorded data at varying concentrations of the fluorescent lipid analogue Abberior STAR Red-DPPE (DPPE: 1,2dipalmitoyl-sn-glycero-3-phosphoethanolamine) diffusing in a fluid supported lipid membrane bilayer (SLB, composed of lipids DOPC, 1,2-dioleoyl-sn-glycero-3-phosphocholine), which is a convenient and well characterised model membrane system, often used as a control sample in STED-FCS experiments ${ }^{22}$. While increasing the lipid analogue concentration and pushing the confocal count rates beyond the conventional FCS range (Figure 4A) did not influence the noise in confocal measurements (Figure 4B, green data points), it - as expected - significantly improved the data quality of the STEDFCS recordings (low nRMSD values, Figure 4B, purple data points; note that for plotting of these, the respective confocal count rates, which limit the experimental conditions for STED recordings, were used as the $x$-value). Also as expected from theory, the values of transit times were smaller in the STED compared to the confocal recordings (due to the reduced observation spot size in the STED mode, here roughly $40 \mathrm{~nm}$ in diameter) and hardly changed with instituted concentrations of the fluorescent probe (i.e., at increased count rate, Figure 4C). Similarly, the observation spot diameters as determined from the recorded FCS data remained constant (Figure 4D, see Materials and Methods for details about its calculation), all in all highlighting the great flexibility and improvement in STEDFCS experiments when employing non-saturating detection electronics.

For simplicity, we demonstrated the above effects at a single STED laser power (150 mW, observation spot diameter of $40 \mathrm{~nm}$ ), but the conclusions held also true for other STED laser powers (and thus observation spot sizes). Similar or even larger improvements of STED-FCS data quality as by increasing the dye concentration were achieved by increasing the excitation laser power (Figure SI 4; note that we, in contrast to the previous data of Atto655, now included an additional decay due to triplet state population in FCS data model, see Materials and Methods and Figure SI 4E). Effects due to saturation 
of the excited states (such as the triplet state) of the fluorescent label started to deteriorate the signal quality and bias the extracted parameter values only at laser powers beyond $25 \mu \mathrm{W}$, supporting the benefits of high count rates for STED-FCS experiments with the excellent dyes available nowadays.

STED-FCS in live cell membranes

Finally, we verified the reliability of STED-FCS measurements at high photon count rates for measurements in living cells. We labelled the plasma membrane of live HeLa cells using the fluorescent lipid analogue Cholesterol-PEG-Abberior STAR Red (Chol-PEG-KK114, Figure 5A), for which previous studies have consistently indicated free diffusion in cellular plasma membranes ${ }^{46}$. The resulting STEDFCS data show low nRMSD values, i.e. low noise levels in the correlation data, which can in the STED microscopy mode be significantly improved to almost confocal quality by increasing either the excitation laser power or concentration of Chol-PEG-KK114 (i.e. total count rate, Figure 5B) without biasing the resulting values of transit times (Figure 5C).

Note that we here measured the diffusion in the apical membrane of HeLa cells, i.e. 5-10 $\mu \mathrm{m}$ above the microscope coverslip (Figure 5A), rather than in the basal membrane as before ${ }^{46}$. This avoids potential biasing effects by the coverslip surface. Yet, penetration through the aqueous cellular environment over such a distance causes spherical aberrations when employing a traditional oilimmersion STED microscope objective (due to the refractive index mismatch between water and oil) ${ }^{47}$, having detrimental effects on STED-FCS experiments (Figure SI 5). Such aberrations can either be corrected for using adaptive optics ${ }^{48,49}$ or employing a water immersion objective $e^{50}$, which shows constant signal levels and performance of for example STED and STED-FCS experiments in a wide range of focal depths (5-100 $\mu \mathrm{m}$ above the coverslip) without the need for depth-dependent readjustments of the correction collar (Figure SI 5). Taking the observation spot size as determined from calibration data (Figure SI 6, diameter of $100 \mathrm{~nm}$ ), we could calculate apparent values of the diffusion coefficient (D) for both the confocal and STED mode (spot diameters of 280 and $100 \mathrm{~nm}$, respectively; see 
Materials and Methods), which were both in the same range $\left(D \approx 0.45 \mu \mathrm{m}^{2} / \mathrm{s}\right.$, Figure $\left.5 D\right)$ as observed before for the basal membrane ${ }^{46}$, highlighting free diffusion and indicating similar diffusion characteristics of the probe in the apical and basal plasma membrane ${ }^{46}$, i.e. negligible bias by the coverslip surface.

A

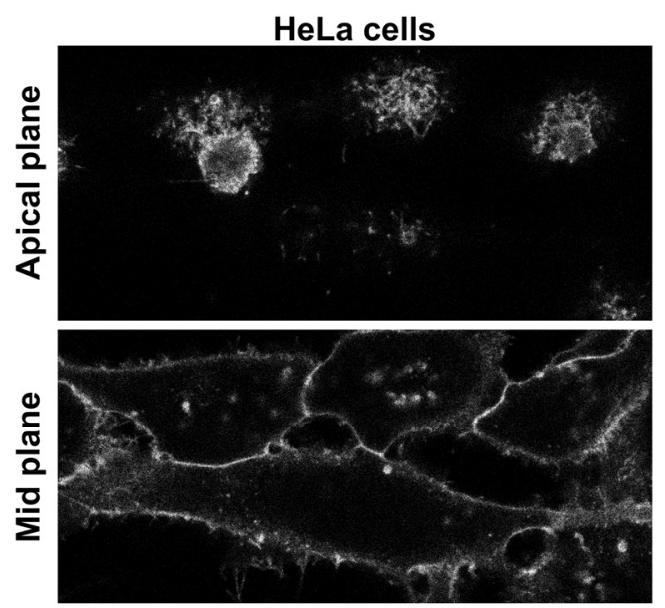

C

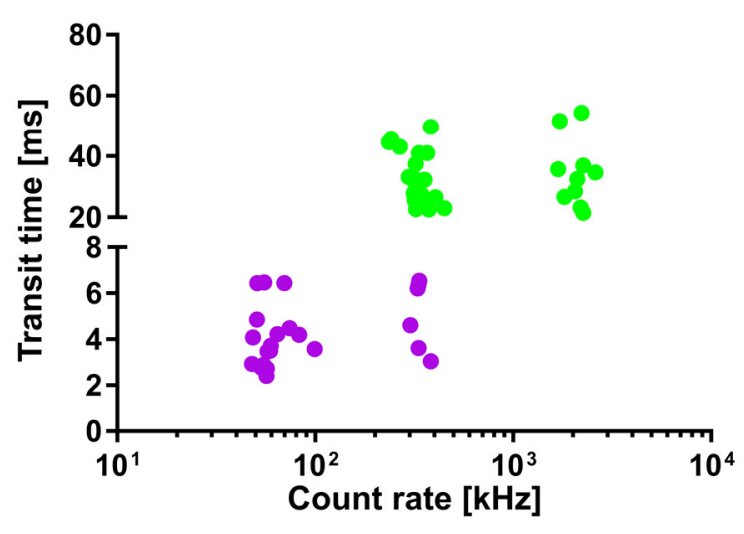

B

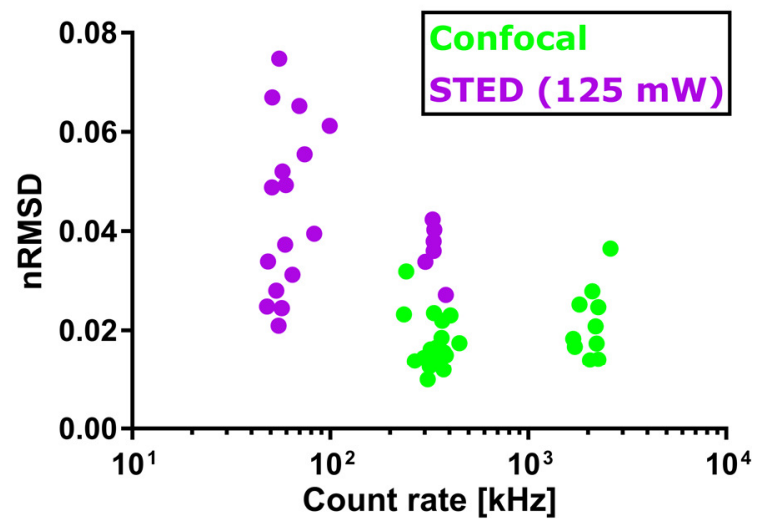

D

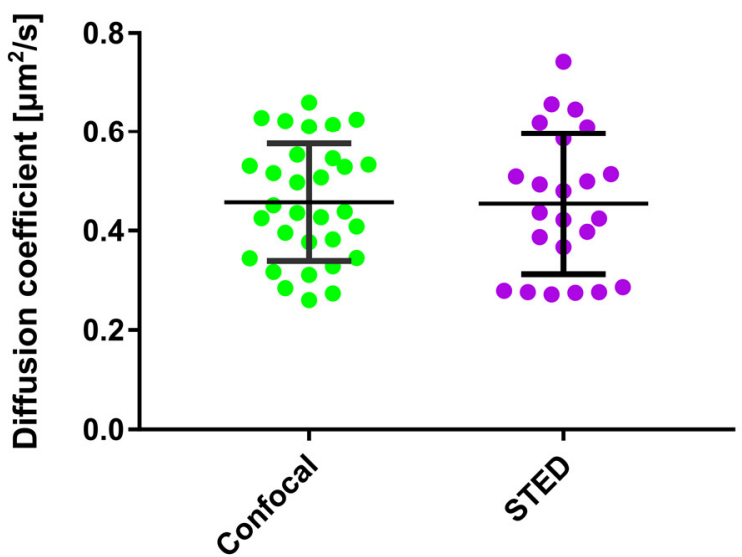

Figure 5: STED-FCS of Abberior STAR-Red-PEG-Cholesterol in membranes of live HeLa cells. A Confocal image of the apical plane (roughly $10 \mu \mathrm{m}$ above the cover slip) and mid plane of HeLa cells, membrane labelled with the fluorescent cholesterol analogue (image sizes $60 \times 27 \mu \mathrm{m}^{2}$ ) B The noise level of FCS data (nRMSD values) and C average fitted transit time for confocal (green) and STED-FCS recordings (125 mW depletion laser power, purple), in the top membrane of HeLa cells, plotted against their respective count rates. D Diffusion coefficient determined from FCS data in confocal and STED mode in the top membrane of the HeLa cells. Every dot represents a single FCS measurement. Excitation laser $2.3 \mu \mathrm{W}$, acquisition time $15 \mathrm{~s}$. 


\section{Conclusions}

We systematically evaluated the reduction in error and bias of FCS measurements recorded at high photon count rates, as enabled by novel detection electronics integrated into a turn-key microscope. We were able to record highly accurate FCS data with detected photon count rates of up to about $10 \mathrm{MHz}$, i.e. dye concentrations up to $1 \mu \mathrm{M}$. This improved performance introduces huge flexibility for performing FCS experiments to measure diffusion or concentration, previously impossible due to limitations in the detection electronics (allowing e.g. only recordings of photon count rates of up to few $\mathrm{MHz}$ ). This now enables: 1) FCS measurements at high dye concentrations for e.g. low-affinity binding assays, 2) the recording of fluctuation data with reduced acquisition times by increasing the excitation power to higher count rates (dye photophysics permitting), 3) performing live-cell experiments in a wide range of expression levels of fluorescently tagged proteins, and 4) optimization of the data quality of STED-FCS recordings over a wide range of observation spot sizes by increasing dye concentration and/or excitation laser power. Using these features we could for example show that cytosolic diffusion of GFP was independent of expression level in live HeLa cells, and that the fluorescent lipid analogue was diffusing freely in the apical membrane similarly as reported before for the basal membrane ${ }^{46}$.

Improved detection instrumentation as the one presented here are becoming increasingly available and will be further optimized, pushing the ease of use of FCS or related measurements, such as fluorescence cross correlation spectroscopy $(\mathrm{FCCS})^{51}$, fluorescence lifetime correlation spectroscopy $(\mathrm{FLCS})^{52}$, number and brightness (N\&B) analysis ${ }^{53}$, or line- and raster-scanning correlation spectroscopy $(\mathrm{RICS})^{12,54}$. In combination with high-throughput methods this could enable the systematic evaluation of overexpression of fluorescent proteins ${ }^{55}$, tracking of dynamically changing diffusion properties, or other previously unattainable applications. 


\section{Acknowledgements}

The authors thank for the funding from MRC Proximity to Discovery funds (MC_PC_16082 (P2D Technologies 2 Therapies)), Marie Skłodowska-Curie Actions (707348; I.U.), Newton-Katip Celebi Institutional Links grant (352333122, E.S.), Medical Research Council (MC_UU_12010/unit programs G0902418 and MC_UU_12025), MRC/BBSRC/EPSRC (MR/K01577X/1), Wolfson Foundation, Wellcome Trust (104924/14/Z/14), Deutsche Forschungsgemeinschaft (Research unit 1905, Excellence Cluster Balance of the Microverse, Collaborative Research Centre 1278 Polytarget), Wellcome Institutional Strategic Support Fund, and Oxford internal funds (EPA Cephalosporin Fund and John Fell Fund), support from the Micron Oxford Advanced Bioimaging Unit (Wellcome Trust funding 107457/Z/15/Z), and Dr. Katharina Reglinski for kindly providing the GFP-SNAP plasmids.

\section{Conflict of interest}

The authors declare no conflict of interest but need to note that MJR, GO and FH are employed at Leica Microsystems that manufactures and sells the SP8 STED FALCON used throughout the study. 


\section{Materials and Methods}

Preparation of dyes in solution

Atto655 NHS-ester (AttoTec), Abberior STAR Red NHS-ester also termed KK114 (Abberior), and 20-nm crimson beads (Thermofisher) were stored at concentration $>10 \mu \mathrm{M}$ and diluted in PBS for measurements.

Preparation of Supported Lipid Bilayers (SLBS)

SLBs were prepared by spin coating as described previously ${ }^{56}$. Briefly, a solution of $1 \mathrm{mg} / \mathrm{mL}$ DOPC (1,2-dioleoyl-sn-glycero-3-phosphocholine, Avanti Polar Lipids) dissolved in 1:2 Methanol:Chloroform was spin coated at $3200 \mathrm{rpm}$ for 45 seconds on a $25 \mathrm{~mm}$ diameter cover slip. The formed lipid film was rehydrated with SLB buffer (150 mM NaCl, $10 \mathrm{mM}$ HEPES, pH 7.4) and washed several times. All cover slips for SLB preparation were piranha cleaned $\left(3: 1, \mathrm{H}_{2} \mathrm{SO}_{4}: \mathrm{H}_{2} \mathrm{O}_{2}\right)$ and stored in water. SLBs were labelled with varying amounts of Abberior STAR Red-DPPE (1,2-dipalmitoyl-sn-glycero-3phosphoethanolamine, Abberior).

\section{Tissue Culture}

HeLa cells were cultured at $37^{\circ} \mathrm{C}$ at $5 \% \mathrm{CO}_{2}$ in high-glucose DMEM (Thermofisher) supplemented with $10 \%$ FBS (Thermofisher), L-Glutamine (Thermofisher) and penicillin/streptomycin (Thermofisher). Cells were seeded onto $35 \mathrm{~mm}$ IBIDI glass bottom dishes coated with fibronectin $(10 \mu \mathrm{g} / \mathrm{mL}$ for $5 \mathrm{~min}$ and washed with PBS) $24 \mathrm{~h}$ prior to performing the measurements.

CHO K1 cells were grown at $37^{\circ} \mathrm{C}$ at $5 \% \mathrm{CO}_{2}$ in DMEM/F12 (Lonza) supplemented with $10 \% \mathrm{FBS}$ and L-Glutamine (both Sigma), and labelled in L15 with a fluorescent lipid analog at room temperature. 
The transfections of GFP-SNAP, cytoplasmic GFP, obtained from Dr. Katharina Reglinski, were performed with Turbofect (ThermoFisher) according to the manufacturer's protocol.

Preparation of Giant Plasma Membrane Vesicles (GPMVs)

GPMVs were prepared as described previously ${ }^{56,57}$. In brief, HeLa cells were cultured as described above but seeded on $35 \mathrm{~mm}$ plastic bottom petri dishes. At a confluency of about $75 \%$, the cells were washed with GPMV buffer ( $150 \mathrm{mM} \mathrm{NaCl}, 2 \mathrm{mM} \mathrm{CaCl} 2,10 \mathrm{mM}$ HEPES, $\mathrm{pH}$ 7.4) and then incubated with $25 \mathrm{mM}$ PFA and $10 \mathrm{mM}$ DTT in GPMV buffer for 2 hours at $37^{\circ} \mathrm{C}$. The GPMV containing supernatant was collected and labelled with Abberior STAR Red-PEG(2kDa)-Cholesterol (Abberior) at a final concentration of $0.5 \mu \mathrm{g} / \mathrm{mL}$ for 10 minutes. GPMVs were non-specifically immobilised on poly-L-lysine (PLL) coated surfaces as described before ${ }^{14}$. All diffusion measurements in GPMVs were performed on the top membrane.

Instrumentation \& microscopy

All experiments were performed on a Leica SP8 STED FALCON (Leica Microsystems) equipped with the HC PL APO 100x/1.40 Oil STED WHITE oil immersion objective lens (SLB measurements) and the HC PL APO 86x/1.20 W motCORR STED WHITE water immersion objective lens with a motorised correction collar (for solution, cytosolic, and apical cell membrane measurements). The STED WHITE 86x water lens has a working distance of $300 \mu \mathrm{m}$, and the motorised correction collar adjusts for refraction index mismatch by optimizing the signal for every sample (coverslip). It is worth noting that a single initial setting of the correction collar was sufficient to correct for varying depth over the investigated range of $100 \mu \mathrm{m}$ (Figure SI 5). We used the 488-nm and 633-nm lines of a white light laser as the excitation source. STED-FCS experiments were performed using a $775 \mathrm{~nm}$ pulsed laser $(80 \mathrm{MHz})$ for depletion with laser powers between 0 and $300 \mathrm{~mW}$ measured at the objective. STED delay time was optimised 
using an SLB sample and minimising the detected photon count rate under high-power STED illumination. The respective notch filters $(775 \mathrm{~nm}, 633 \mathrm{~nm}$ or $488 \mathrm{~nm}$ ) were used for emission clean up. For all measurements with constant excitation power we stayed below saturation intensity (by checking proportionality of excitation laser power and fluorescence intensity) as triplet pumping may result in an additional source of deviations ${ }^{58}$. Fluctuations in laser intensities, which in some other studies reflected in a pronounced correlation component with decay times on the order of seconds and had to be corrected for $^{33}$, were not observable in our case - all FCS curves converged to 0 (see examples in Figures 1, SI 1, and SI 7).

All FCS experiments were performed using the hybrid detectors (HyD-SMDs), featuring very short dead times, and FALCON electronics allowing acquisition of TCSPC data at photon count rates of up to 80 Mcps per detection channel without the necessity for corrections, becoming comparable to or exceeding the repetition rates of commonly used pulsed excitation lasers. This implementation is based on sampling the signal from the pulsed laser and detectors using fast FPGA electronics and applying pattern matching to the resulting bitstreams, producing as output the photon arrival times with a resolution of $97 \mathrm{ps}$ and dead time $<1.5 \mathrm{~ns}$, at $\mathrm{GHz}$ sampling rates (for more technical details, please see the Leica Falcon Application Note ${ }^{59}$ ). Though certain other detector types such as avalanche photodiodes (APDs) offer 2-3-fold higher quantum efficiency in the far-red part of the spectrum compared to HyD-SMDs, their dead times are typically around 30 ns, i.e. 20-fold longer. The hereemployed technology thus offers the highest currently achievable overall count rates, which are now on pair with the repetition rate of the excitation laser. Nevertheless, future developments towards increasing the detectors' quantum efficiencies will allow further benefits, e.g. the use of lower excitation laser powers to achieve similarly high count rates or superior signal-to-noise ratios at high STED powers.

Measurement times ranged as indicated from 10 to 60 seconds. Only for the lifetime measurements we used $40 \mathrm{MHz}$ pulsing of the white light laser for excitation. 


\section{Data analysis}

Correlation, time trace cropping, gating and fitting was performed using the built-in routines in LAS-X (Leica Microsystems). Time gates were applied in STED-FCS to remove confocal or laser scattering contributions and therefore improve resolution (see Figure SI 7), while not affecting confocal measurements (Figure SI 7; marginal deterioration of signal-to-noise or slightly larger spread of the fitted parameters were barely noticable). Solution and cytoplasmic GFP data were fitted with a free 3D diffusion model including offset and a triplet component ${ }^{44}$ as appropriate (triplet correlation decay time of GFP $40 \mu$ s with relative amplitude fixed to $14 \%$, Atto655 no triplet population ${ }^{60}$, Abberior STAR Red triplet correlation decay time $5 \mu$ s with triplet amplitudes within 5-10\%; crimson beads triplet correlation decay time 10-100 $\mu$ s with triplet amplitudes 3-5 \%). SLB, GPMV and cell membrane data were fitted with a $2 \mathrm{D}$ anomalous diffusion model ${ }^{61}$ (including offset and triplet time for Abberior STAR Red-PEG-Chol or -DPPE: $5 \mu$ s with relative amplitude around $10 \%$, and up to $30 \%$ at the highest excitation powers). The parameter optimisation was performed using a Levenberg-Marquardt nonlinear least-squares minimisation method with data-points weighted by their standard deviations, which were estimated from variations in ACFs calculated from sub-sections of the intensity time trace $^{27}$. Some representative autocorrelation curves together with their fits, non-weighted fitting residuals, and reduced $\chi^{2}$ values ${ }^{27}$ are displayed in Figures SII and SI8.

As a measure of data quality and curve smoothness, defining the precision of the extracted fitted parameters, nRMSD values were calculated by taking the root-mean-square difference between the measured FCS curve and its fit up to the transit time, and normalised to the fitted amplitude. Such measure yielded similar values as the experimental standard deviation of the autocorrelation curves ${ }^{27}$ normalised to the amplitude (Figure SI1 and SI8; for easier comparison to nRMSD, the main data quality assessment tool throughout this work, we there display non-weighted residuals, but plot also standard deviations to put the residuals in the right perspective relevant to fitting). Smooth data- 
points at longer lag times were excluded, to avoid the influence of model miss-fitting in extreme conditions applied in this study, e.g. when curves were distorted due to saturation or photobleaching effects at the highest excitation powers, or by random bright transits occurring in cells. The constructed measure nRMSD thus correlates well with the relative standard deviation of the fitted transit times (Figure SI 2D).

For concentration estimation, we assumed a confocal volume of $1 \mathrm{fL}$. Given that the correlation amplitude relates to the inverse average number of particles in the focal volume, concentrations can be estimated ${ }^{10}$. For STED-FCS experiments the diffusion coefficient was calculated as described before ${ }^{22}$ using the following formula:

$$
D=\frac{\omega^{2}}{8 \cdot \ln (2) \cdot \tau_{D}}
$$

Where $D$ is the apparent diffusion coefficient (in confocal or STED), $\omega$ refers to the full width half max (FWHM) of the observation spot (in confocal or STED) and $\tau_{D}$ to the transit time extracted from the FCS fit. The FWHM was determined by confocal and STED imaging of fluorescent beads ( $20 \mathrm{~nm}$ crimson beads). Using the assumption that the fluorescently labelled lipids diffuse freely in a SLB, the FWHM can be calculated as a function of STED power using the following equation ${ }^{22}$ :

$$
\omega(\text { STED })=\omega(\text { confocal }) \cdot \sqrt{ }\left(\frac{\tau_{D, S T E D}}{\tau_{D, \text { confocal }}}\right)
$$

Note that we used the top membrane of immobilised GPMVs labelled with Abberior STAR Red-PEGCholesterol to determine the FWHM far away from the surface using a water immersion objective. 


\section{References}

(1) Magde, D.; Elson, E.; Webb, W. W. Thermodynamic Fluctuations in a Reacting System-

Measurement by Fluorescence Correlation Spectroscopy. Phys. Rev. Lett. 1972, 29, 705-708.

(2) Elson, E. L. Fluorescence Correlation Spectroscopy: Past, Present, Future. Biophys. J. 2011, $101,2855-2870$.

(3) Ehrenberg, M.; Rigler, R. Rotational Brownian Motion and Fluorescence Intensify Fluctuations. Chem. Phys. 1974, 4, 390-401.

(4) Cebecauer, M.; Amaro, M.; Jurkiewicz, P.; Sarmento, M. J.; Šachl, R.; Cwiklik, L.; Hof, M. Membrane Lipid Nanodomains. Chem. Rev. 2018, 118, 11259-11297.

(5) Sezgin, E.; Levental, I.; Mayor, S.; Eggeling, C. The Mystery of Membrane Organization: Composition, Regulation and Roles of Lipid Rafts. Nat. Rev. Mol. Cell Biol. 2017, 18, 361-374.

(6) Di Bona, M.; Mancini, M. A.; Mazza, D.; Vicidomini, G.; Diaspro, A.; Lanzanò, L. Measuring Mobility in Chromatin by Intensity-Sorted FCS. Biophys. J. 2019, 116, 987-999.

(7) Lanzanò, L.; Scipioni, L.; Di Bona, M.; Bianchini, P.; Bizzarri, R.; Cardarelli, F.; Diaspro, A.; Vicidomini, G.; Bona, M. Di; Bianchini, P.; Bizzarri, R.; Di Bona, M.; Bianchini, P.; Bizzarri, R.; Cardarelli, F.; Diaspro, A.; Vicidomini, G.; Bona, M. Di; Bianchini, P. et al. Measurement of Nanoscale Three-Dimensional Diffusion in the Interior of Living Cells by STED-FCS. Nat. Commun. 2017, 8, 65.

(8) Eggeling, C.; Hellriegel, C. Editorial. Methods 2018, 140-141, 1-2.

(9) Jacobson, K.; Liu, P.; Lagerholm, B. C. The Lateral Organization and Mobility of Plasma Membrane Components. Cell 2019, 177, 806-819.

(10) Lackowicz, J. Principles of Fluorescence Spectroscopy, Third.; Lakowicz, J. R., Ed.; Springer US: Boston, MA, 2006. 
(11) Koppel, D. E. Statistical Accuracy in Fluorescence Correlation Spectroscopy. Phys. Rev. A 1974, $10,1938-1945$.

(12) Ruan, Q.; Cheng, M. A.; Levi, M.; Gratton, E.; Mantulin, W. W. Spatial-Temporal Studies of Membrane Dynamics: Scanning Fluorescence Correlation Spectroscopy (SFCS). Biophys. J. 2004, 87, 1260-1267.

(13) Ries, J.; Chiantia, S.; Schwille, P. Accurate Determination of Membrane Dynamics with LineScan FCS. Biophys. J. 2009, 96, 1999-2008.

(14) Schneider, F.; Waithe, D.; Lagerholm, B. C.; Shrestha, D.; Sezgin, E.; Eggeling, C.; Fritzsche, M. Statistical Analysis of Scanning Fluorescence Correlation Spectroscopy Data Differentiates Free from Hindered Diffusion. ACS Nano 2018, 12, 8540-8546.

(15) Rossow, M. J.; Sasaki, J. M.; Digman, M. a; Gratton, E. Raster Image Correlation Spectroscopy in Live Cells. Nat. Protoc. 2010, 5, 1761-1774.

(16) Sankaran, J.; Manna, M.; Guo, L.; Kraut, R.; Wohland, T. Diffusion, Transport, and Cell Membrane Organization Investigated by Imaging Fluorescence Cross-Correlation Spectroscopy. Biophys. J. 2009, 97, 2630-2639.

(17) Jin, W.; Simsek, M. F.; Pralle, A. Quantifying Spatial and Temporal Variations of the Cell Membrane Ultra-Structure by BimFCS. Methods 2018, 140-141, 151-160.

(18) Hebert, B.; Costantino, S.; Wiseman, P. W. Spatiotemporal Image Correlation Spectroscopy (STICS) Theory, Verification, and Application to Protein Velocity Mapping in Living CHO Cells. Biophys. J. 2005, 88, 3601-3614.

(19) Schwille, P.; Meyer-Almes, F. J.; Rigler, R. Dual-Color Fluorescence Cross-Correlation Spectroscopy for Multicomponent Diffusional Analysis in Solution. Biophys. J. 1997, 72, 18781886. 
(20) Wawrezinieck, L.; Rigneault, H.; Marguet, D.; Lenne, P.-F. Fluorescence Correlation Spectroscopy Diffusion Laws to Probe the Submicron Cell Membrane Organization. Biophys. J. $2005,89,4029-4042$.

(21) Kusumi, A.; Nakada, C.; Ritchie, K.; Murase, K.; Suzuki, K.; Murakoshi, H.; Kasai, R. S.; Kondo, J.; Fujiwara, T. Paradigm Shift of the Plasma Membrane Concept from the Two-Dimensional Continuum Fluid to the Partitioned Fluid: High-Speed Single-Molecule Tracking of Membrane Molecules. Annu. Rev. Biophys. Biomol. Struct. 2005, 34, 351-378.

(22) Sezgin, E.; Schneider, F.; Galiani, S.; Urbančič, I.; Waithe, D.; Lagerholm, B. C.; Eggeling, C. Measuring Nanoscale Diffusion Dynamics in Cellular Membranes with Super-Resolution STED-FCS. Nat. Protoc. 2019, 14, 1054-1083.

(23) Moran, U. Average concentration of a protein in cell; BNID 104520 https://bionumbers.hms.harvard.edu/bionumber.aspx?id=104520 (accessed Sep 24, 2019).

(24) Kask, P.; Günther, R.; Axhausen, P. Statistical Accuracy in Fluorescence Fluctuation Experiments. Eur. Biophys. J. 1997, 25, 163-169.

(25) Saffarian, S.; Elson, E. L. Statistical Analysis of Fluorescence Correlation Spectroscopy: The Standard Deviation and Bias. Biophys. J. 2003, 84, 2030-2042.

(26) Qian, H. On the Statistics of Fluorescence Correlation Spectroscopy. Biophys. Chem. 1990, 38, $49-57$.

(27) Wohland, T.; Rigler, R.; Vogel, H. The Standard Deviation in Fluorescence Correlation Spectroscopy. Biophys. J. 2001, 80, 2987-2999.

(28) Waithe, D.; Schneider, F.; Chojnacki, J.; Clausen, M. P.; Shrestha, D.; de la Serna, J. B.; Eggeling, C. Optimized Processing and Analysis of Conventional Confocal Microscopy Generated Scanning FCS Data. Methods 2018, 140-141, 62-73. 
(29) Böhmer, M.; Wahl, M.; Rahn, H.-J.; Erdmann, R.; Enderlein, J. Time-Resolved Fluorescence Correlation Spectroscopy. Chem. Phys. Lett. 2002, 353, 439-445.

(30) Isbaner, S.; Karedla, N.; Ruhlandt, D.; Stein, S. C.; Chizhik, A.; Gregor, I.; Enderlein, J. DeadTime Correction of Fluorescence Lifetime Measurements and Fluorescence Lifetime Imaging. Opt. Express 2016, 24, 9429-9445.

(31) Manley, S.; Gillette, J. M.; Patterson, G. H.; Shroff, H.; Hess, H. F.; Betzig, E.; LippincottSchwartz, J. High-Density Mapping of Single-Molecule Trajectories with Photoactivated Localization Microscopy. Nat. Methods 2008, 5, 155-157.

(32) Eggeling, C.; Hilbert, M.; Bock, H.; Ringemann, C.; Hofmann, M.; Stiel, A. C.; Andresen, M.; Jakobs, S.; Egner, A.; Schönle, A.; Hell, S. W. Reversible Photoswitching Enables SingleMolecule Fluorescence Fluctuation Spectroscopy at High Molecular Concentration. Microsc. Res. Tech. 2007, 70, 1003-1009.

(33) Laurence, T. A.; Ly, S.; Bourguet, F.; Fischer, N. O.; Coleman, M. A. Fluorescence Correlation Spectroscopy at Micromolar Concentrations without Optical Nanoconfinement. J. Phys.

Chem. B 2014, 118, 9662-9667.

(34) Laurence, T. A.; Weiss, S. Analytical Chemistry: How to Detect Weak Pairs. Science. January 31, 2003, pp 667-668.

(35) Blom, H.; Kastrup, L.; Eggeling, C. Fluorescence Fluctuation Spectroscopy in Reduced Detection Volumes. Curr. Pharm. Biotechnol. 2006, 7, 51-66.

(36) Foquet, M.; Korlach, J.; Zipfel, W. R.; Webb, W. W.; Craighead, H. G. Focal Volume Confinement by Submicrometer-Sized Fluidic Channels. Anal. Chem. 2004, 76, 1618-1626.

(37) Wenger, J.; Rigneault, H.; Dintinger, J.; Marguet, D.; Lenne, P. F. Single-Fluorophore Diffusion in a Lipid Membrane over a Subwavelength Aperture. J. Biol. Phys. 2006, 32, 18-21. 
(38) Leutenegger, M.; Gösch, M.; Perentes, A.; Hoffmann, P.; Martin, O. J. F.; Lasser, T. Confining the Sampling Volume for Fluorescence Correlation Spectroscopy Using a Sub-Wavelength Sized Aperture. Opt. Express 2006, 14, 956.

(39) Kinkhabwala, A. A.; Yu, Z.; Fan, S.; Moerner, W. E. Fluorescence Correlation Spectroscopy at High Concentrations Using Gold Bowtie Nanoantennas. Chem. Phys. 2012, 406, 3-8.

(40) Manzo, C.; van Zanten, T. S.; Garcia-Parajo, M. F. Nanoscale Fluorescence Correlation Spectroscopy on Intact Living Cell Membranes with NSOM Probes. Biophys. J. 2011, 100, L8L10.

(41) Regmi, R.; Winkler, P. M.; Flauraud, V.; Borgman, K. J. E.; Manzo, C.; Brugger, J.; Rigneault, H.; Wenger, J.; García-Parajo, M. F. Planar Optical Nanoantennas Resolve Cholesterol-Dependent Nanoscale Heterogeneities in the Plasma Membrane of Living Cells. Nano Lett. 2017, 17, 6295-6302.

(42) Kastrup, L.; Blom, H.; Eggeling, C.; Hell, S. W. Fluorescence Fluctuation Spectroscopy in Subdiffraction Focal Volumes. Phys. Rev. Lett. 2005, 94, 178104.

(43) Barzan, M.; Hajiesmaeilbaigi, F. Investigation the Concentration Effect on the Absorption and Fluorescence Properties of Rhodamine 6G Dye. Optik (Stuttg). 2018, 159, 157-161.

(44) Widengren, J.; Mets, U.; Rigler, R. Fluorescence Correlation Spectroscopy of Triplet States in Solution: A Theoretical and Experimental Study. J. Phys. Chem. 1995, 99, 13368-13379.

(45) Schönle, A.; Von Middendorff, C.; Ringemann, C.; Hell, S. W.; Eggeling, C. Monitoring Triplet State Dynamics with Fluorescence Correlation Spectroscopy: Bias and Correction. Microsc. Res. Tech. 2014, 77, 528-536.

(46) Honigmann, A.; Mueller, V.; Ta, H.; Schoenle, A.; Sezgin, E.; Hell, S. W.; Eggeling, C. Scanning STED-FCS Reveals Spatiotemporal Heterogeneity of Lipid Interaction in the Plasma Membrane of Living Cells. Nat. Commun. 2014, 5, 5412. 
(47) Hell, S.; Reiner, G.; Cremer, C.; Stelzer, E. H. K. Aberrations in Confocal Fluorescence

Microscopy Induced by Mismatches in Refractive Index. J. Microsc. 1993, 169, 391-405.

(48) Antonello, J.; Burke, D.; Booth, M. J. Aberrations in Stimulated Emission Depletion (STED)

Microscopy. Opt. Commun. 2017, 404, 203-209.

(49) Barbotin, A.; Galiani, S.; Urbančič, I.; Eggeling, C.; Booth, M. J. Adaptive Optics Allows STED-

FCS Measurements in the Cytoplasm of Living Cells. Opt. Express 2019, 27, 23378.

(50) Heine, J.; Wurm, C. A.; Keller-Findeisen, J.; Schönle, A.; Harke, B.; Reuss, M.; Winter, F. R.;

Donnert, G. Three Dimensional Live-Cell STED Microscopy at Increased Depth Using a Water Immersion Objective. Rev. Sci. Instrum. 2018, 89, 053701.

(51) Bacia, K.; Schwille, P. Practical Guidelines for Dual-Color Fluorescence Cross-Correlation Spectroscopy. Nat. Protoc. 2007, 2, 2842-2856.

(52) Ghosh, A.; Karedla, N.; Thiele, J. C.; Gregor, I.; Enderlein, J. Fluorescence Lifetime Correlation Spectroscopy: Basics and Applications. Methods 2018, 140-141, 32-39.

(53) Digman, M. A.; Dalal, R.; Horwitz, A. F.; Gratton, E. Mapping the Number of Molecules and Brightness in the Laser Scanning Microscope. Biophys. J. 2008, 94, 2320-2332.

(54) Digman, M. A.; Brown, C. M.; Sengupta, P.; Wiseman, P. W.; Horwitz, A. R.; Gratton, E. Measuring Fast Dynamics in Solutions and Cells with a Laser Scanning Microscope. Biophys. J. 2005, 89, 1317-1327.

(55) Wachsmuth, M.; Conrad, C.; Bulkescher, J.; Koch, B.; Mahen, R.; Isokane, M.; Pepperkok, R.; Ellenberg, J. High-Throughput Fluorescence Correlation Spectroscopy Enables Analysis of Proteome Dynamics in Living Cells. Nat. Biotechnol. 2015, 33, 384-389.

(56) Schneider, F.; Waithe, D.; Clausen, M. P.; Galiani, S.; Koller, T.; Ozhan, G.; Eggeling, C.; Sezgin, E. Diffusion of Lipids and GPI-Anchored Proteins in Actin-Free Plasma Membrane Vesicles 
Measured by STED-FCS. Mol. Biol. Cell 2017, 28, 1507-1518.

(57) Sezgin, E.; Kaiser, H.-J.; Baumgart, T.; Schwille, P.; Simons, K.; Levental, I. Elucidating Membrane Structure and Protein Behavior Using Giant Plasma Membrane Vesicles. Nat. Protoc. 2012, 7, 1042-1051.

(58) Enderlein, J.; Gregor, I.; Patra, D.; Fitter, J. Art and Artefacts of Fluorescence Correlation Spectroscopy. Curr. Pharm. Biotechnol. 2004, 5, 155-161.

(59) Alvarez, L. A. J.; Widzgowski, B.; Ossato, G.; van den Broek, B.; Jalink, K.; Kuschel, L.; Roberti, M. J.; Hecht, F. Application Note: SP8 FALCON: a novel concept in fluorescence lifetime imaging enabling video-rate confocal FLIM. Nat. Methods 2019.

(60) Enderlein, J.; Gregor, I. Using Fluorescence Lifetime for Discriminating Detector Afterpulsing in Fluorescence-Correlation Spectroscopy. Rev. Sci. Instrum. 2005, 76, 1-5.

(61) Sanabria, H.; Kubota, Y.; Waxham, M. N. Multiple Diffusion Mechanisms Due to Nanostructuring in Crowded Environments. Biophys. J. 2007, 92, 313-322. 


\section{Supporting figures}

High excitation power (38.8 $\mu \mathrm{W})$

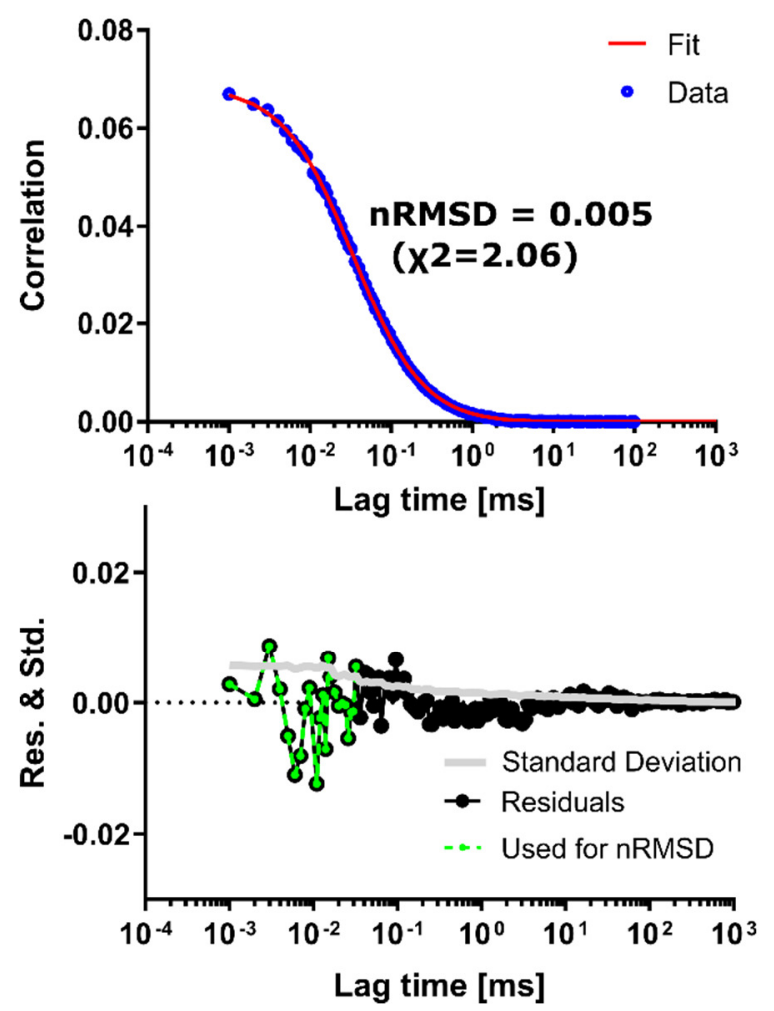

Low excitation power $(0.8 \mu W)$
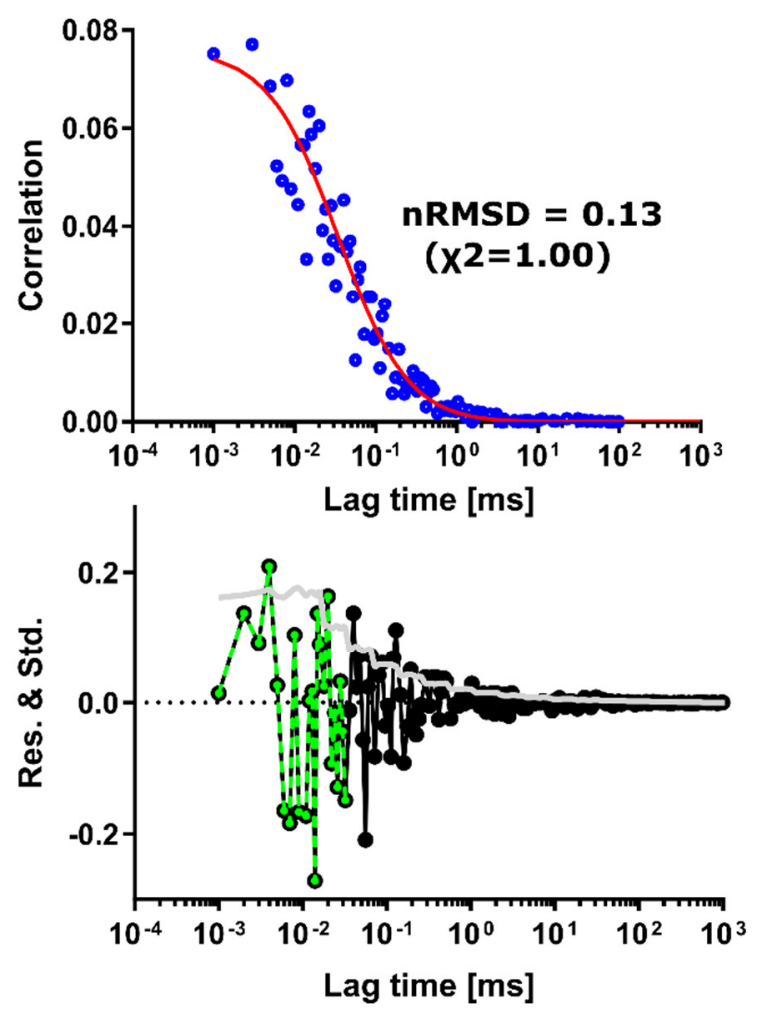

Figure SI 1: Calculation of nRMSD values from the (non-weighted) fitting residuals as a measure of the noise level in autocorrelation curves. FCS data of $5 \mathrm{nM}$ Atto655 in aqueous solution acquired at high and low excitation power (38.8 and $0.8 \mu \mathrm{W}$, blue data-points in left and right panels, respectively) with respective fits (red curves) and fitting residuals (black) and experimental standard deviations of the autocorrelation at every lag time (grey), both normalised by the amplitude, below. The residuals highlighted in green (up to the lag time of the fitted transit time) where used for the calculation of the nRMSD value; the lower the nRMSD, the better the data quality. In fitting, though, the residuals were weighted by the respective standard deviations for the determination of the reduced $\chi^{2}$-values as the minimisation metric (see Methods). 
A

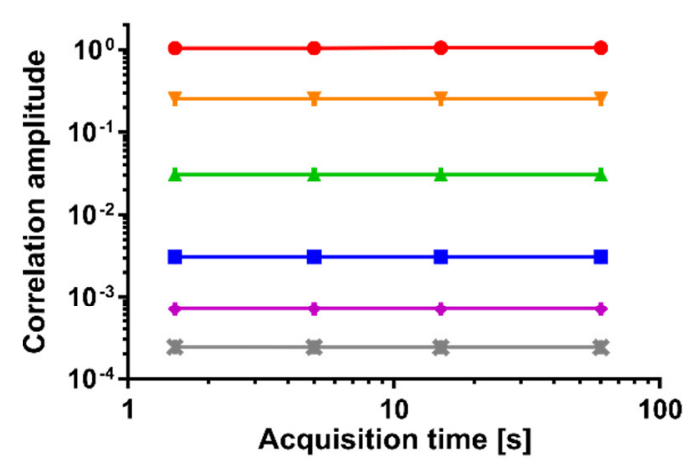

C

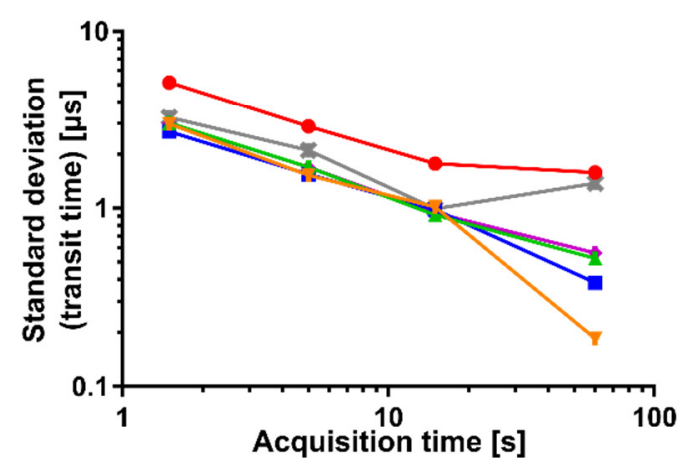

B

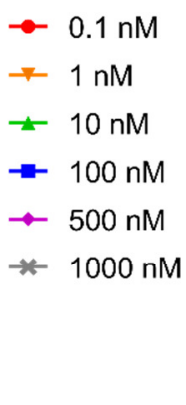

D

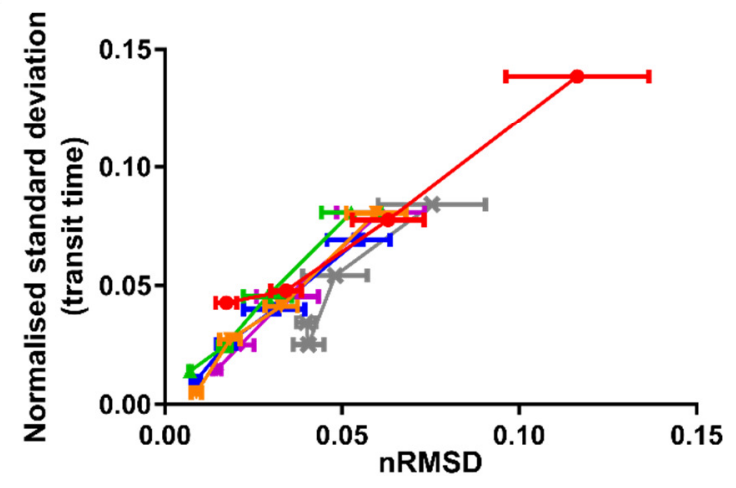

Figure SI 2: Effect of acquisition time on FCS performance at different concentrations of Atto655 in aqueous solution A The fitted correlation amplitude, B transit time, and C standard deviation of the fitted transit time, plotted against the acquisition time. D Relative standard deviation of the fitted transit times against the FCS noise level (nRMSD). Measurements were recorded at excitation laser power $15.4 \mu \mathrm{W}$ and acquisition time 60 $s$, and then chopped into shorter intervals, as indicated. 
A

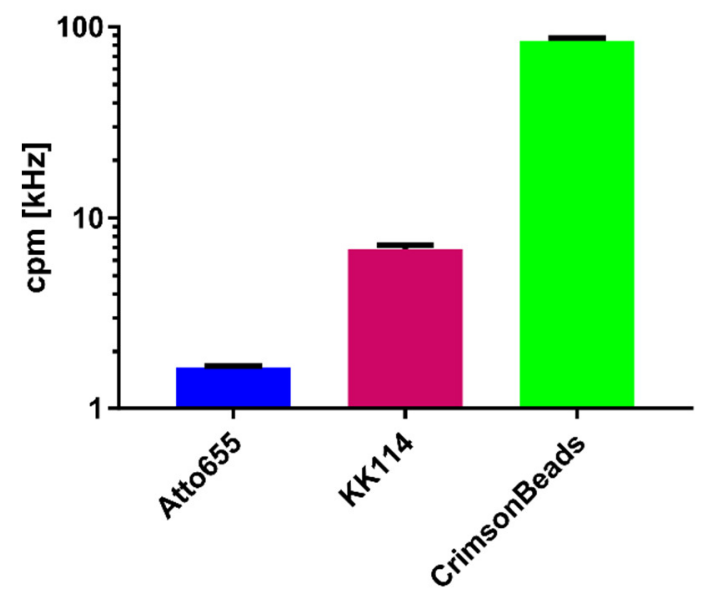

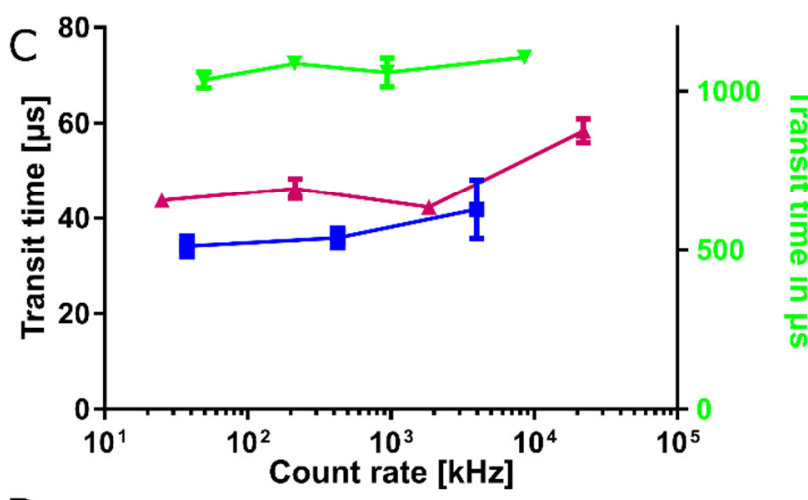

D

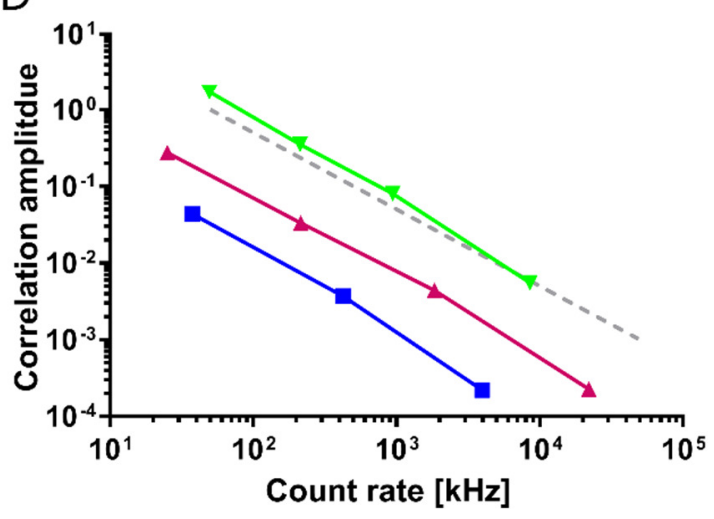

B
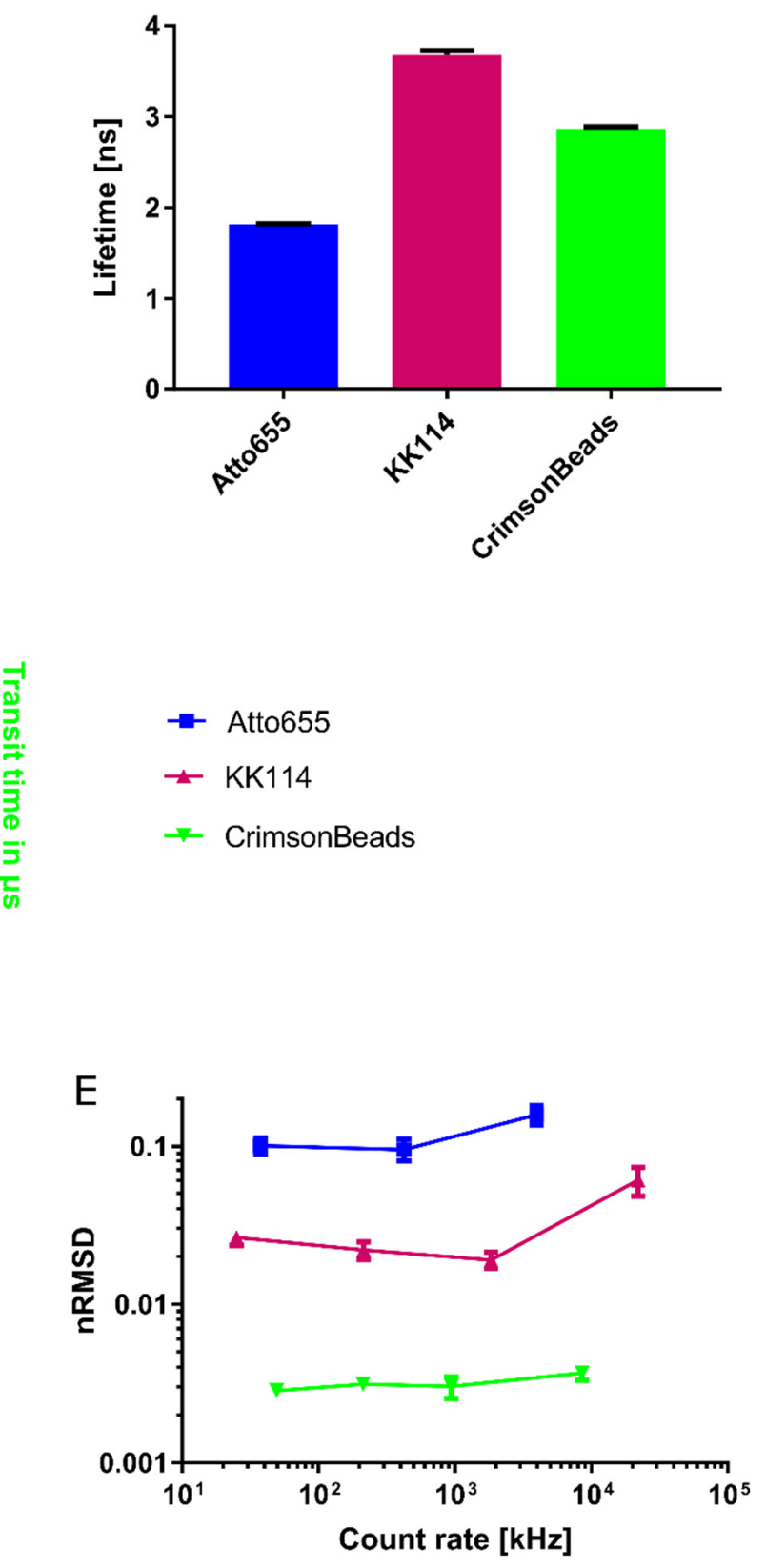

Figure SI 3: Influence of molecular brightness and fluorescence lifetime of dyes on performance in FCS at different count rates. A Counts per molecule and B fluorescence lifetime from single exponential fits to TCSPC histograms, for aqueous solutions of Atto655, KK114 (Abberior STAR Red) and Crimson Beads C Fitted transit time, D Correlation amplitude, and E FCS noise level (nRMSD) obtained for various concentrations of Atto655 $(0.01,0.1$, and $1 \mu \mathrm{M}), \mathrm{KK} 114(0.01,0.1,1$, and $10 \mu \mathrm{M})$ and Crimson Beads $(1: 2000,1: 500,1: 100$, and 1:10 dilutions of the purchased stock solution), resulting in different count rates. Excitation laser power $1.5 \mu \mathrm{W}$, acquisition time $60 \mathrm{~s}$, data-points represent averages and standard deviations of at least 3 measurements. The grey dashed line in D represents a linear relationship. Average nRMSD values from panel E were used for the inset in Figure 2E. 
A

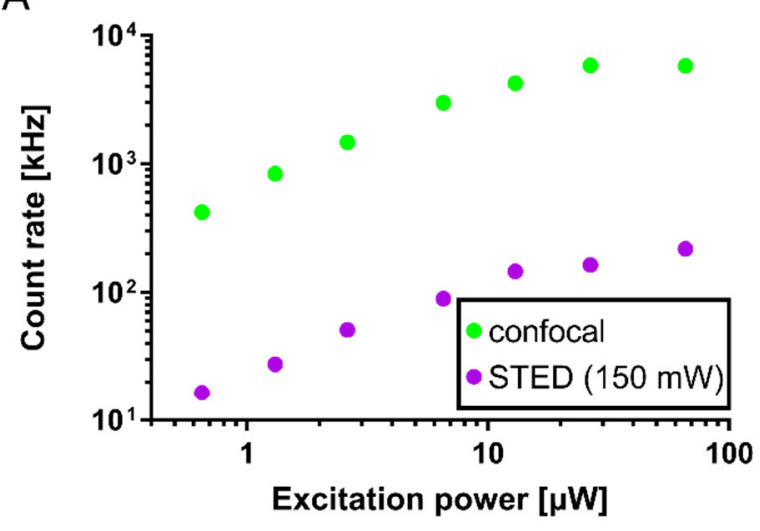

C

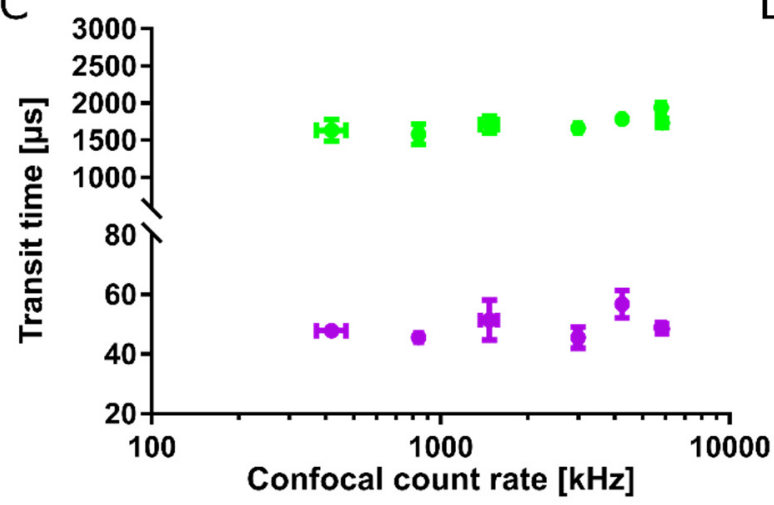

$E$

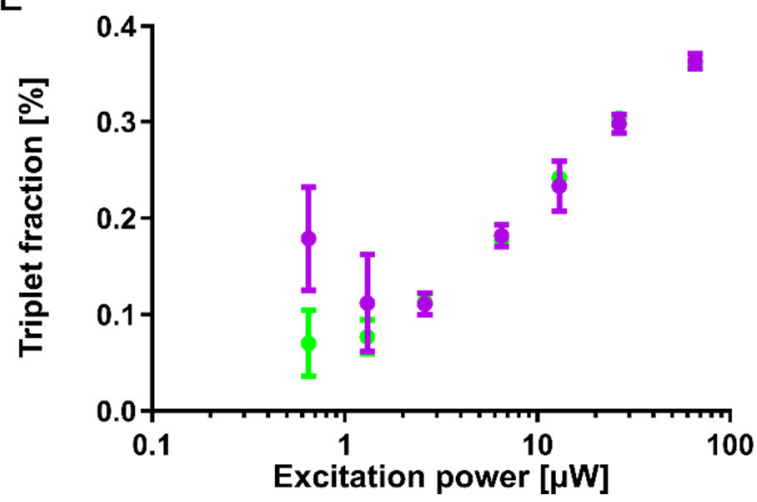

B

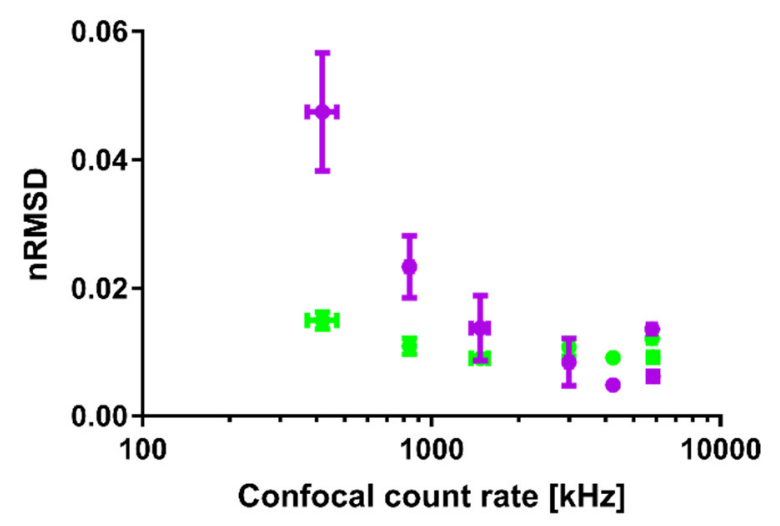

$\mathrm{D}$

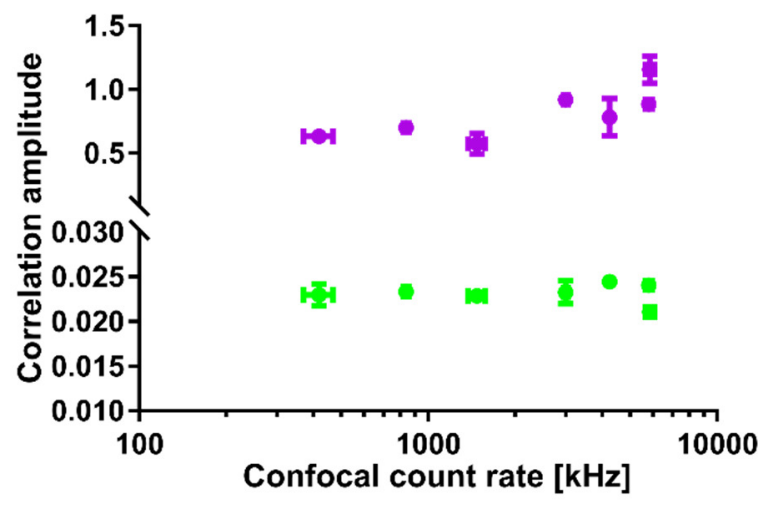

$\mathrm{F}$

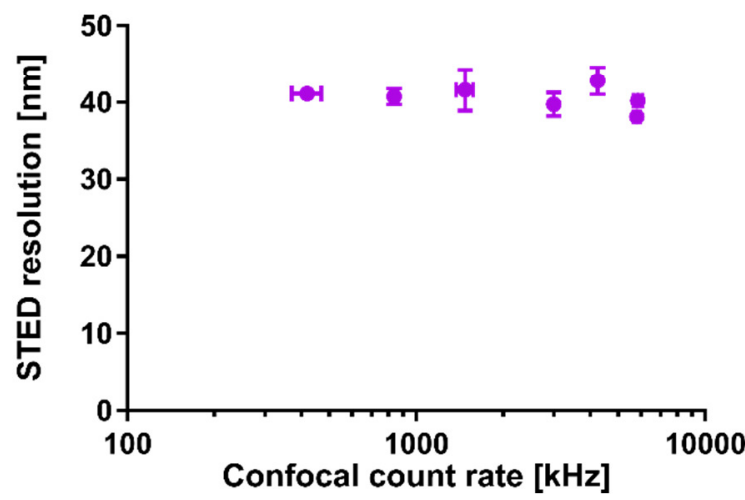

Figure SI 4: STED-FCS measurements of fluorescent Abberior STAR Red- DPPE in a DOPC SLB - acquired at varying excitation laser powers from $0.7 \mu \mathrm{W}$ to $66 \mu \mathrm{W}$. A Change in confocal (green) and STED (purple) count rate when varying the excitation power. B The FCS noise level (nRMSD), and fit values of $\mathbf{C}$ transit times, $\mathbf{D}$ correlation amplitude, $\mathbf{E}$ triplet fractions, and $\mathbf{F}$ apparent STED resolution plotted against confocal count rate for confocal (green) and STED recordings (purple). Acquisition time $15 \mathrm{~s}$, depletion laser power $150 \mathrm{~mW}$. Data-points represent averages and standard deviations of at least 3 measurements. 


\section{$20 \mathrm{~nm}$ Crimson Beads in solution}

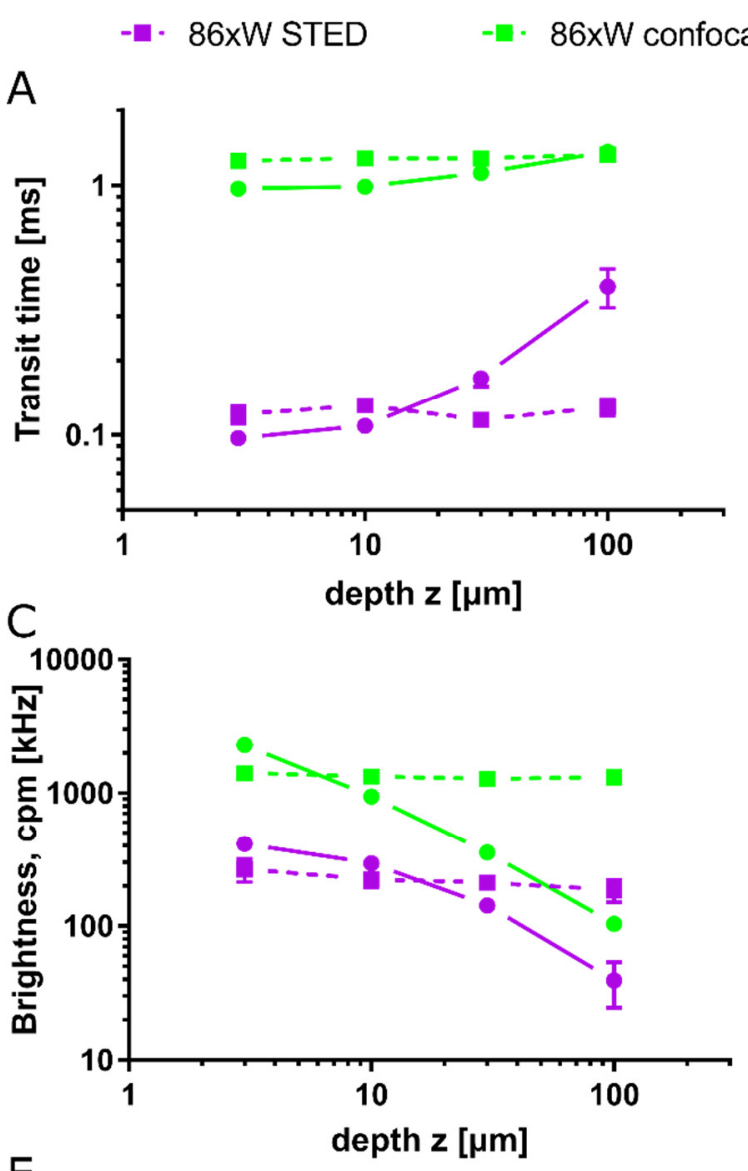

$E$

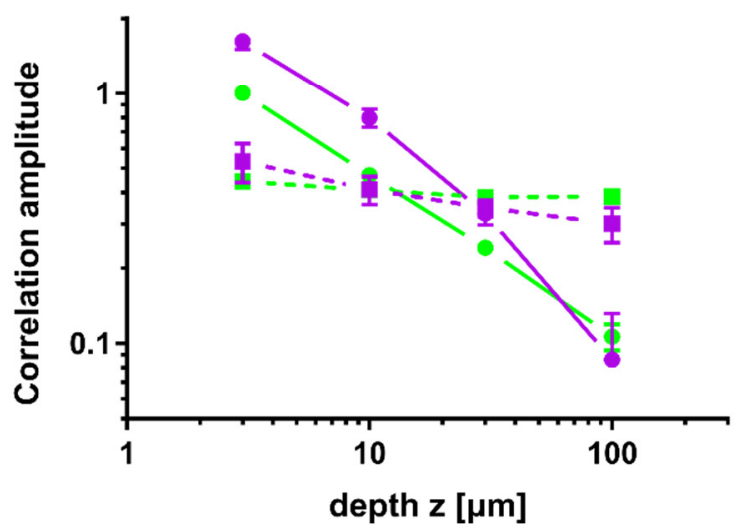

SiR-SNAP in cytoplasm of GPMVs
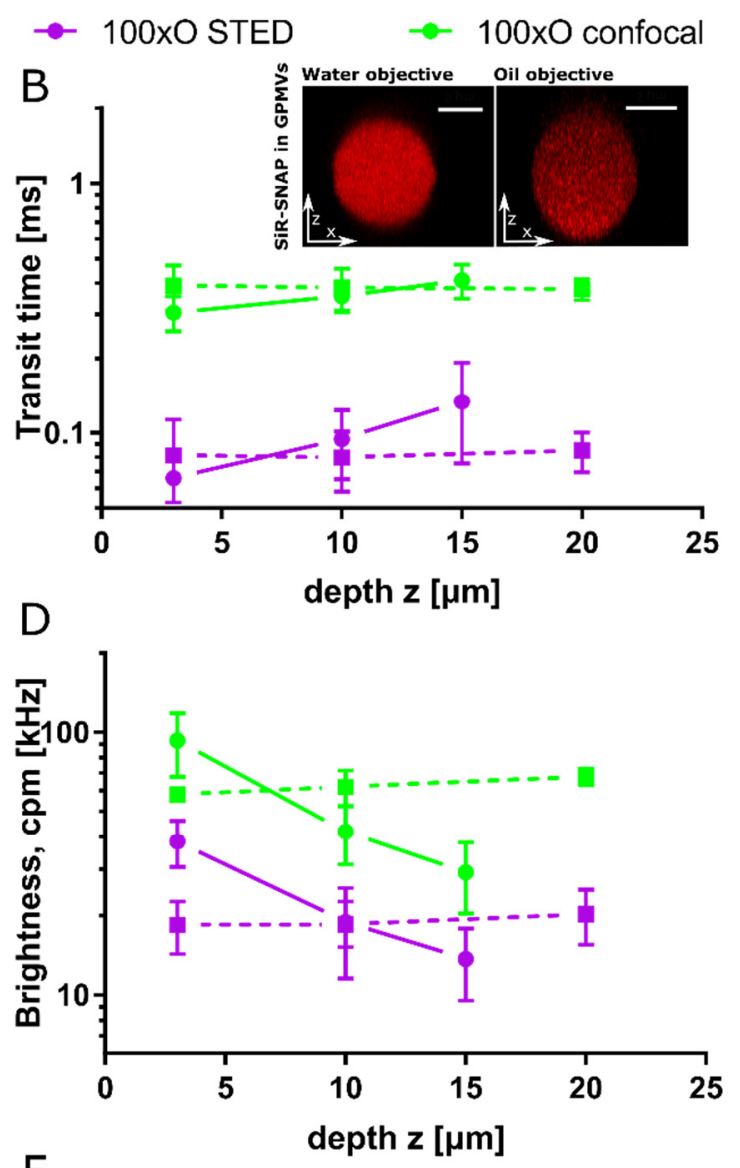

$\mathrm{F}$

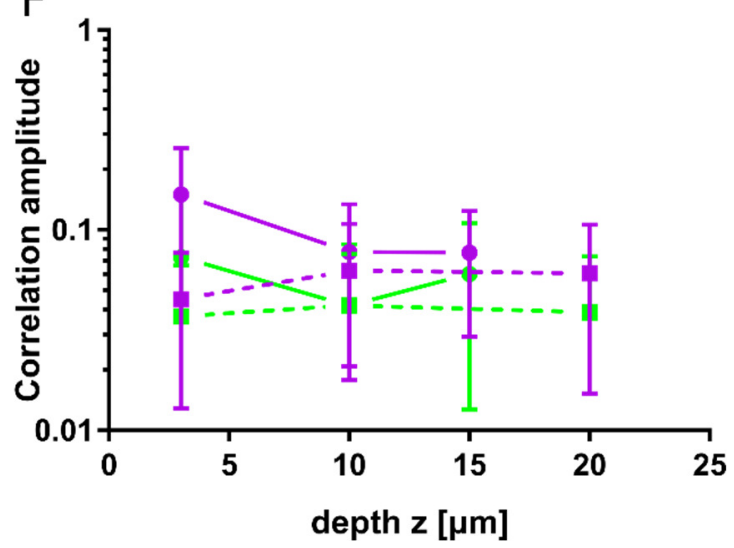

Figure SI 5: Performance of (STED-)FCS depends on objective and depth. (STED-)FCS experiments at different focal depth (z) for $20 \mathrm{~nm}$ Crimson beads in water (left panel A, C, E) and SiR-SNAP in cytoplasm of immobilised CHO GPMVs (right panel B,D,F) using a 100x oil- (circles, solid lines) and 86x water-immersion objective (squares, dashed lines). A,B The transit times, $\mathbf{C , D}$ molecular brightness (cpm) and $\mathbf{E}, \mathbf{F}$ correlation amplitude determined from measurements at different focal depths in confocal (green) and STED mode (purple). Excitation laser power $8.9 \mu \mathrm{W}$, depletion laser powers 140 and $56 \mathrm{~mW}$ for crimson beads and SiR-SNAP, respectively, acquisition time $20 \mathrm{~s}$. All data points are averages and standard deviations from at least three measurements. The inset in B shows confocal images of axial cross-sections of CHO GPMVs with SiR-SNAP in the cytoplasm. 


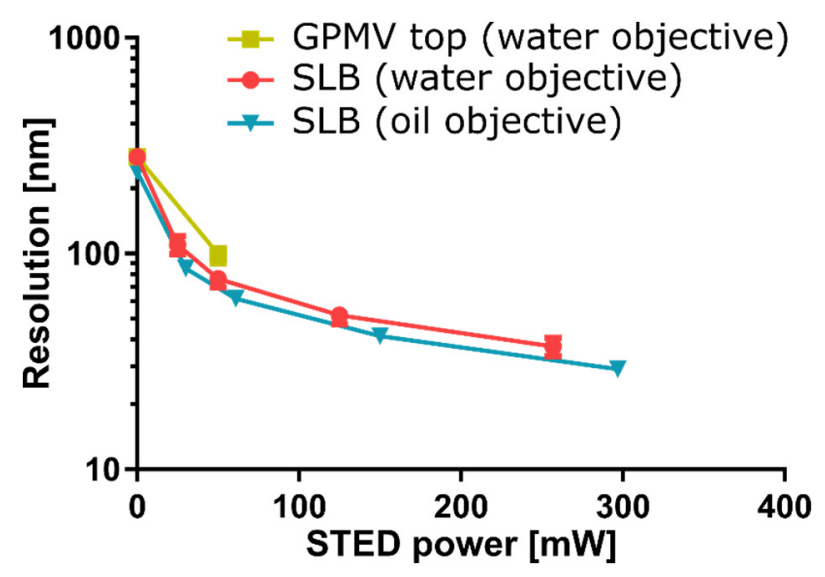

Figure SI 6: STED-FCS resolution calibration using SLBs (Abberior STAR Red-DPPE in DOPC) with oil- (blue) and water-immersion (orange) objectives, compared to the resolution obtained from the top membrane of $\mathrm{CHO}$ GPMVs (labelled with freely diffusing Abberior STAR Red-PEG-Cholesterol with a water-immersion objective (yellow)). Excitation power was $2.3 \mu \mathrm{W}$ and depletion power was $125 \mathrm{~mW}$, acquisition time $15 \mathrm{~s}$. From at least three measurements per condition, the average value and their standard deviation are shown. 
A

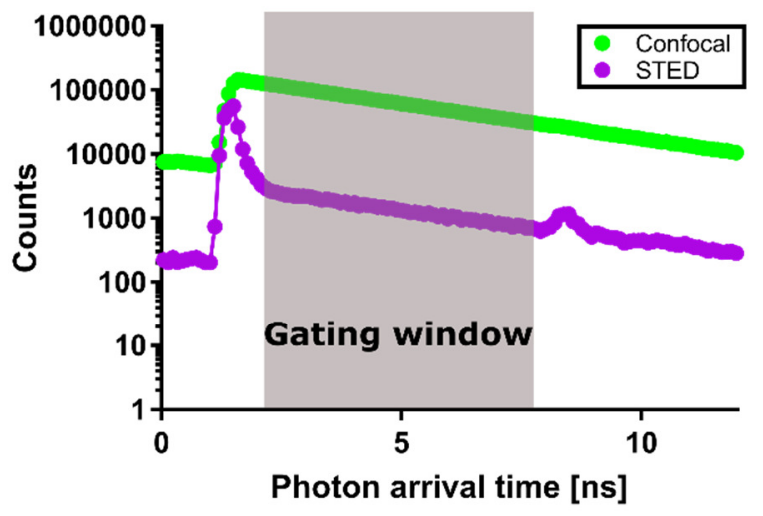

C

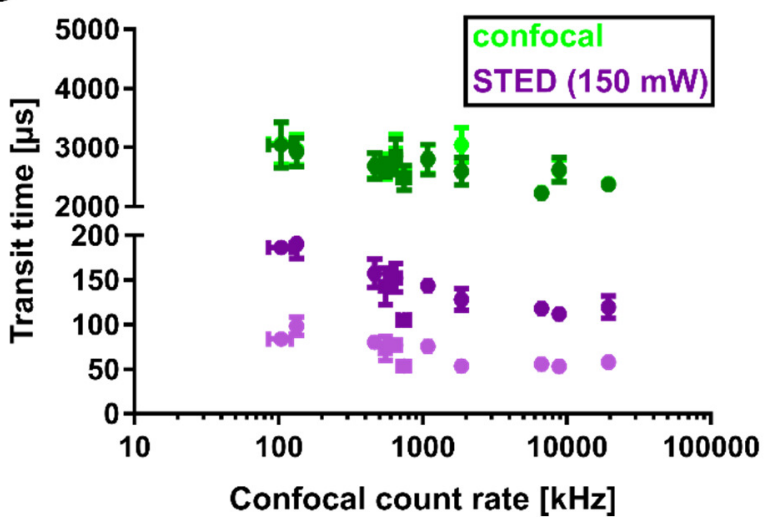

B

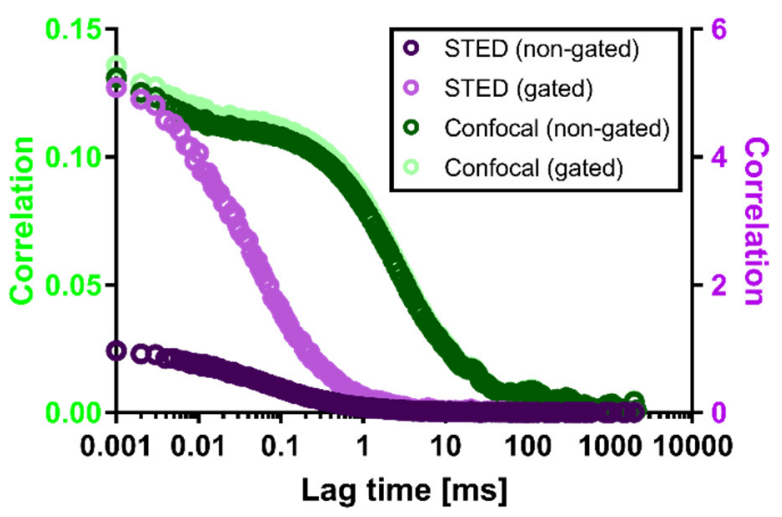

D

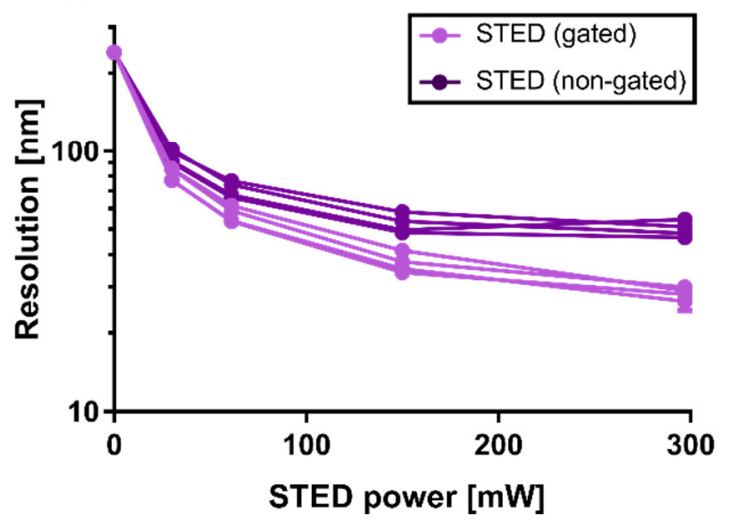

Figure SI 7: Influence of time gating in STED-FCS A Time correlated single photon counting (TCSPC) histograms of photon arrival times for an exemplary confocal (green) and STED-FCS measurement (purple, $150 \mathrm{~mW}$ depletion power) on a DOPC SLB labelled with Abberior STAR Red - DPPE. The gating window (grey area) was chosen to exclude confocal contributions to the STED-FCS correlation and to exclude the bump from the white light laser reflection in our system (note that it is present with the same absolute intensity in both datasets, but less visible for confocal data with higher fluorescence counts due to logarithmic scaling of the $y$-axis). B Exemplary correlation curves, and $\mathbf{C}$ average fitted transit times for confocal (green) and STED recordings (purple), analysed with and without gating applied (lighter and darker symbols, respectively). D Apparent resolution (i.e., diameter of the effective observation spot) as calculated from confocal and STED transit times for gated (light purple) and non-gated (dark purple) data acquired at different STED laser powers. Measurements of $15 \mathrm{~s}$ were acquired at $2.3 \mu \mathrm{W}$ excitation laser power. In panels $\mathrm{C}$ and $\mathrm{D}$, average and standard deviation of three measurements are shown. 

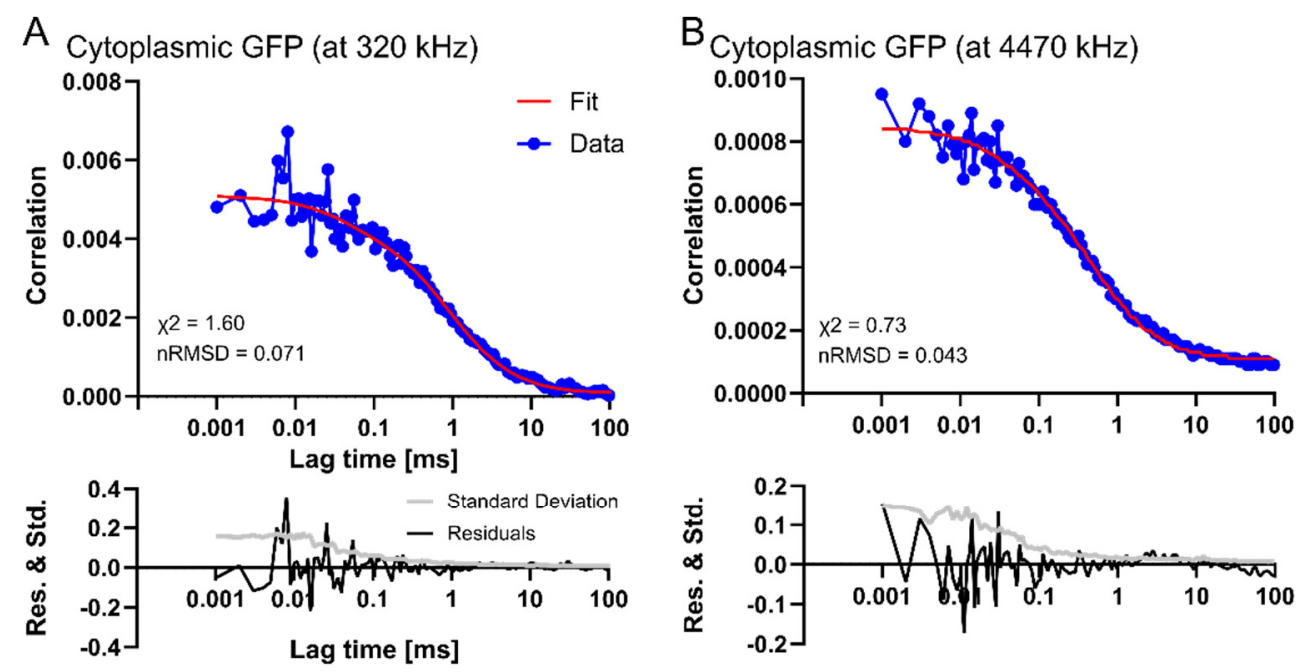

C SLB confocal (at $450 \mathrm{kHz})$

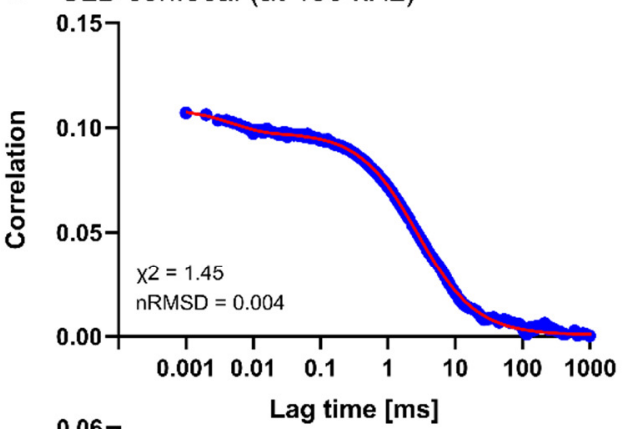

D SLB STED (at $240 \mathrm{kHz})$
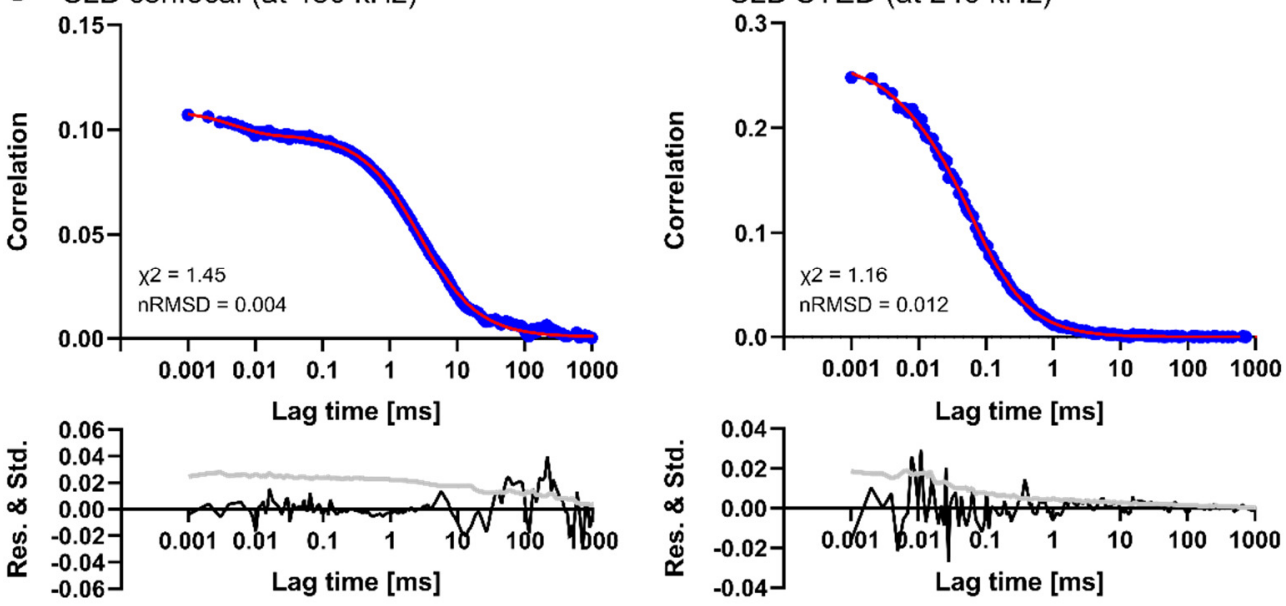

E KK114-PEG-Chol in HeLa (confocal)

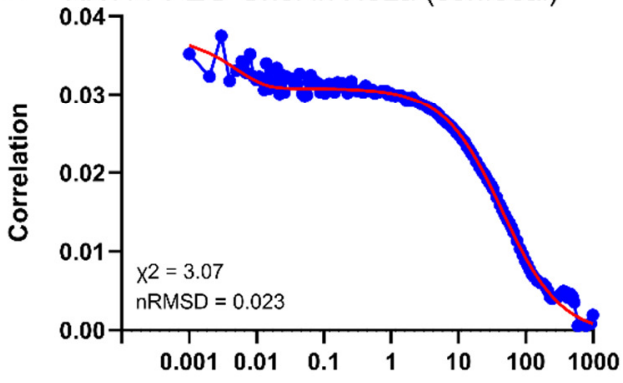

F KK114-PEG-Chol in HeLa (STED)
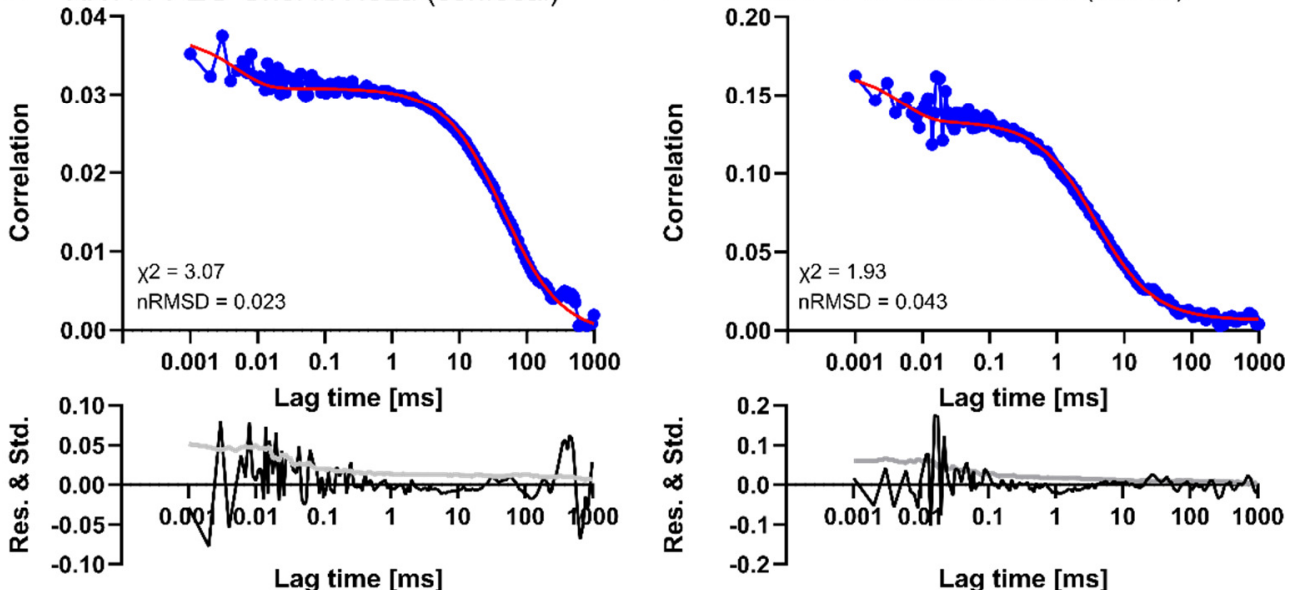

Figure SI 8: Representative FCS data with their fits (top panels), non-weighted fitting residuals (Res., black lines) and experimental standard deviation of the autocorrelation (Std., grey lines) plotted in the bottom panels, both normalised to the amplitude of the respective FCS curve. A and B: Fitting of a 3D diffusion model with a triplet state to correlation curves obtained from cytoplasmic GFP at high and low count rates, respectively (data from Figure 3). C and D: Fitting of a 2D diffusion model with a triplet state to confocal and STED data of KK114-DPPE diffusion in an SLB composed of DOPC (data from Figure 4). E and F: Fitting of a 2D diffusion model with a triplet 

available under aCC-BY 4.0 International license.

state to correlation data obtained from KK114-PEG Cholesterol diffusing in the plasma membrane of live HeLa cells in confocal and STED acquisition mode (data from Figure 5). Non-weighted residuals were used to determine our signal quality measure nRMSD, whereas the standard deviations were taken into account to calculate the reduced $\chi^{2}$ as the minimisation metric in fitting (see Methods for details). 